\title{
WISE J072003.20-084651.2: AN OLD AND ACTIVE M9.5 + T5 SPECTRAL BINARY 6 pc FROM THE SUN*
}

\author{
Adam J. Burgasser ${ }^{1,2,8}$, Michaël Gillon ${ }^{3}$, Carl Melis $^{1}$, Brendan P. Bowler ${ }^{4,9,10}$, Eric L. Michelsen ${ }^{1}$, \\ Daniella Bardalez Gagliuffi ${ }^{1}$, Christopher R. Gelino ${ }^{5,6}$, E. Jehin ${ }^{2}$, L. Delrez ${ }^{2}$, J. Manfroid ${ }^{2}$, and Cullen H. Blake ${ }^{7}$ \\ ${ }^{1}$ Center for Astrophysics and Space Science, University of California San Diego, La Jolla, CA, 92093, USA; aburgasser@ucsd.edu \\ 2 Instituto de Astrofísica de Canarias (IAC), La Laguna, Tenerife, Spain \\ ${ }^{3}$ Institute of Astrophysics and Géophysique, Université of Liège, allée du 6 Août, 17, B-4000 Liège, Belgium \\ ${ }^{4}$ California Institute of Technology, Division of Geological and Planetary Sciences, 1200 East California Boulevard, Pasadena, CA 91101, USA \\ ${ }^{5}$ NASA Exoplanet Science Institute, Mail Code 100-22, California Institute of Technology, 770 South Wilson Avenue, Pasadena, CA 91125 , USA \\ ${ }^{6}$ Infrared Processing and Analysis Center, MC 100-22, California Institute of Technology, Pasadena, CA 91125, USA \\ ${ }^{7}$ Department of Physics and Astronomy, University of Pennsylvania, Philadelphia, PA 19104, USA \\ Received 2014 August 13; accepted 2014 October 15; published 2015 February 19
}

\begin{abstract}
We report observations of the recently discovered, nearby late-M dwarf WISE J072003.20-084651.2. New astrometric measurements obtained with the TRAPPIST telescope improve the distance measurement to $6.0 \pm 1.0$ pc and confirm the low tangential velocity $\left(3.5 \pm 0.6 \mathrm{~km} \mathrm{~s}^{-1}\right)$ reported by Scholz. Low-resolution optical spectroscopy indicates a spectral type of M9.5 and prominent $\mathrm{H} \alpha$ emission $\left(\left\langle\log _{10} L_{H \alpha} / L_{\mathrm{bol}}\right\rangle=-4.68 \pm 0.06\right)$, but no evidence of subsolar metallicity or Li I absorption. Near-infrared spectroscopy reveals subtle peculiarities that can be explained by the presence of a T5 binary companion, and high-resolution laser guide star adaptive optics imaging reveals a faint $(\Delta H=4.1)$ candidate source 0 !' $14(0.8 \mathrm{AU})$ from the primary. With high-resolution optical and near-infrared spectroscopy, we measure a stable radial velocity of $+83.8 \pm 0.3 \mathrm{~km} \mathrm{~s}^{-1}$, indicative of old disk kinematics and consistent with the angular separation of the possible companion. We measure a projected rotational velocity of $v \sin i=8.0 \pm 0.5 \mathrm{~km} \mathrm{~s}^{-1}$ and find evidence of low-level variabilty $(\sim 1.5 \%)$ in a 13 day TRAPPIST light curve, but cannot robustly constrain the rotational period. We also observe episodic changes in brightness $(1 \%-2 \%)$ and occasional flare bursts (4\%-8\%) with a $0.8 \%$ duty cycle, and order-of-magnitude variations in $\mathrm{H} \alpha$ line strength. Combined, these observations reveal WISE J0720-0846 to be an old, very low-mass binary whose components straddle the hydrogen burning minimum mass, and whose primary is a relatively rapid rotator and magnetically active. It is one of only two known binaries among late M dwarfs within $10 \mathrm{pc}$ of the Sun, both of which harbor a mid T-type brown dwarf companion. We show that while this specific configuration is rare ( $\lesssim 1.6 \%$ probability), roughly $25 \%$ of binary companions to late-type $\mathrm{M}$ dwarfs in the local population are likely low-temperature $\mathrm{T}$ or $\mathrm{Y}$ brown dwarfs.
\end{abstract}

Key words: binaries: spectroscopic - binaries: visual - brown dwarfs - stars: individual (WISE J072003.20084651.2) - stars: low-mass - stars: magnetic field

\section{INTRODUCTION}

Stars and brown dwarfs in the immediate Solar Neighborhood $(d<10$ pc; Reid et al. 1995, 2008; Henry et al. 2006; Cruz et al. 2007) are ideal targets for detailed investigations of the structural, atmospheric and populative properties of these objects. This is particularly true for the very lowest-mass (VLM) and lowest-luminosity dwarfs-the late-M, -L, -T, and -Y dwarfs-whose recent discovery has been facilitated by wide-area red and infrared imaging surveys (e.g., 2MASS, DENIS, SDSS, UKIDSS, WISE) and multi-epoch red and infrared astrometry (e.g., LSPM, SuperCOSMOS, AllWISE). Yet, despite their apparent brightness and high proper motion,

\footnotetext{
* Some of the data presented herein were obtained at the W. M. Keck Observatory, which is operated as a scientific partnership among the California Institute of Technology, the University of California and the National Aeronautics and Space Administration. The Observatory was made possible by the generous financial support of the W. M. Keck Foundation.

${ }^{8}$ Visiting Astronomer at the Infrared Telescope Facility, which is operated by the University of Hawaii under Cooperative Agreement no. NNX-08AE38A with the National Aeronautics and Space Administration, Science Mission Directorate, Planetary Astronomy Program.

${ }^{9}$ Caltech Joint Center for Planetary Astronomy Fellow.

${ }^{10}$ Visiting Astronomer, Kitt Peak National Observatory, National Optical Astronomy Observatory, which is operated by the Association of Universities for Research in Astronomy (AURA) under cooperative agreement with the National Science Foundation.
}

up to $\sim 20 \%$ of our nearest $(<20 \mathrm{pc})$ VLM neighbors remain "missing," particularly toward the Galactic plane (Reid et al. 2004; Lépine 2005; Henry et al. 2006), as exemplified by the very recent discovery of the third (Luhman 16AB; Luhman 2013) and fourth (WISE J085510.83-071442.5; Kirkpatrick et al. 2014; Luhman 2014) closest systems to the Sun, comprised of L, T, and Y dwarfs. Several very low-temperature $\mathrm{T}$ and Y dwarfs (Artigau et al. 2010; Lucas et al. 2010; Cushing et al. 2011; Kirkpatrick et al. 2011; Scholz et al. 2011; Bihain et al. 2013) and even M dwarfs (e.g., Teegarden et al. 2003; Hambly et al. 2004; Deacon et al. 2005a; Scholz et al. 2005; Henry et al. 2006; Scholz 2014) have also been uncovered within $5 \mathrm{pc}$ of the Sun in the past decade.

One of the most recent nearby discoveries is WISE J072003.20-084651.2 (hereafter WISE J0720-0846), a candidate $M / L$ dwarf identified by Scholz (2014) in the WISE survey. With a parallax distance measurement of only $7.0 \pm 1.9$ pc, this source had been "hiding" in the Galactic plane, its

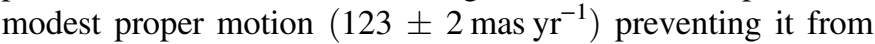
being picked up in earlier astrometric surveys. Scholz (2014) estimated a photometric classificaiton of $\mathrm{M} 9 \pm 1$, which was confirmed in near-infrared spectroscopy reported by Kirkpatrick et al. (2014). Given its proximity to the Sun, WISE J0720-0846 is an important new system for investigating the M 
dwarf/L dwarf and star/brown dwarf transitions at high spatial, spectral and temporal resolution.

In this article, we report new observations that confirm WISE J0720-0846 to be a nearby (6 pc), late-M dwarf, and show it to have old disk kinematics, magnetic activity (including flares), and rapid rotation. We further identify it as a spectral binary system with a T-type brown dwarf companion, possibly resolved at a separation of $\sim 0.8 \mathrm{AU}$ and estimated orbital period of 2-4 yr. In Section 2 we describe our observations of the system, including low- and high-resolution optical and near-infrared spectroscopy, high-resolution imaging, and red optical monitoring. In Section 3 we analyze these data, determining the optical and near-infrared classifications, improved astrometry, spatial kinematics, rotation, magnetic emission and limits on the photometric variability of this source. In Section 4 we identify the binary nature of WISE J0720-0846, both as a spectral binary and a potentially resolved system, and characterize its orbital properties from imaging and multi-epoch radial velocity measurements. In Section 5 we review the physical properties of this source, and examine it in the context of multiplicity among late $M$ dwarfs in the immediate vicinity of the Sun. Results are summarized in Section 6.

\section{OBSERVATIONS}

\subsection{Low-resolution Red Optical Spectroscopy}

WISE J0720-0846 was observed on 2013 December 30 (UT) with the Ritchey-Chretien Spectrograph (RC Spec) on the Kitt Peak National Observatory's $4 \mathrm{~m}$ Mayall telescope. RC Spec was equipped with the T2KA CCD, and we used the BL420 grating blazed at $7800 \AA$ (first order) and GG-475 order blocking filter with the $1.15 \times 98^{\prime \prime}$ slit aligned north-south, yielding spectral data spanning 6300-9000 $\AA$ at a resolving power of $\sim 3 \AA$. The source was observed near transit at an airmass of 1.32 in a single integration of $1000 \mathrm{~s}$. We also observed the spectrophotometric standard HR 3454 (Hamuy et al. 1992) at an airmass of 1.15 for flux calibration, as well as flat field and HeNeAr arc lamps. Data were reduced using custom Interactive Data Language (IDL) routines which corrected images for bad pixels and cosmic rays, performed bias-subtraction, and flat-fielding. After sky background subtraction and spectral extraction (summing along the spatial axis), we calibrated the wavelength scale to air wavelengths using the arclamp spectrum, and corrected for throughput losses using the standard star observation. No correction for telluric absorption was attempted. The reduced spectrum is shown in Figure 1 and discussed further in Section 3.

\subsection{Low-resolution Near-infrared Spectroscopy}

A low-resolution near-infrared spectrum of WISE J07200846 was obtained on 2013 December 5 (UT) using the SpeX spectrograph mounted on the $3 \mathrm{~m}$ NASA Infrared Telescope Facility (IRTF; Rayner et al. 2003). Conditions were clear with $1^{\prime \prime}$ seeing at $J$-band. We used the SpeX prism mode with the 0.5 slit aligned with the parallactic angle, yielding $0.8-2.45$ $\mu \mathrm{m}$ spectra with an average resolution $\lambda \Delta \lambda \approx 120$. Six exposures of $30 \mathrm{~s}$ each were obtained at an airmass of 1.14 , followed by observations of the A0 V star HD 56525 $(V=7.19)$ at an airmass of 1.20. HeNeAr arc lamps and quartz lamp exposures were also obtained for dispersion and pixel response calibration. Data were reduced using the
SpeXtool package version 3.4 (Vacca et al. 2003; Cushing et al. 2004) following standard procedures for point-source extraction. These data are similar to those reported by Kirkpatrick et al. (2014). The reduced spectrum is shown in Figure 2 and discussed further in Section 4.

\subsection{High Resolution Optical Spectroscopy}

WISE J0720-0846 was observed with the Hamilton echelle spectrograph (Vogt 1987) on the Lick Observatory Shane $3 \mathrm{~m}$ telescope over seven nights between 2013 December 21 and 2014 February 26 (UT; Table 1). Conditions varied from clear to overcast, with seeing between $0.1 / 8-1 / 5$. We used the 640 $\mu \mathrm{m}$ slit, 31.5 lines $\mathrm{mm}^{-1}$ grating, and UBK (crown glass) cross-dispersing prisms to obtain $3500-10000 \AA$ spectroscopy over 107 orders, at a resolution of $\lambda / \Delta \lambda=62,000$ as measured from the FWHM of single arclines in lamp spectra. Multiple integrations of 40 minutes were obtained while the source was above an airmass of 2.0. On each night, halogen lamp flat field frames were obtained for pixel response calibration, and TiAr arclamp spectra were obtained for wavelength calibration. The M4 radial velocity standard GJ 251 (Nidever et al. 2002) was observed each night following WISE J0720-0846 for radial velocity calibration.

Data were reduced in the IRAF environment ${ }^{11}$ following Churchill (1995). Briefly, data were bias subtracted, flatfielded, box-car extracted, and wavelength calibrated using Ti and $\mathrm{Ar}$ lines from arclamp exposures (Pakhomov \& Zhao 2013). These data are discussed further in Section 3.

\subsection{High Resolution Near-infrared Spectroscopy}

High resolution near-infrared $K$-band spectra of WISE J0720-0846 were obtained with the NIRSPEC echelle spectrograph on the Keck II telescope (McLean et al. 2000) on four nights, 2014 January 19 and 20, March 10 and April 12 (UT; Table 2). For each night we used the N7 order-sorting filter and 0.432 wide slit to obtain 2.00-2.39 $\mu \mathrm{m}$ spectra over orders 32-38 with $\lambda / \Delta \lambda=20,000\left(\Delta v=15 \mathrm{~km} \mathrm{~s}^{-1}\right)$ and dispersion of $0.315 \AA$ pixel $^{-1}$. Two dithered exposures of 360-600 s each were obtained, along with observations of the nearby A0 V stars HD 65102 (night $1, V=6.83$ ) and HD 65158 (nights $2-4, V=7.16$ ). Flat field and dark frames were obtained at the start of each night for detector calibration.

Data were reduced using a modified version of the REDSPEC package, which took the rectified images produced by that routine and optimally extracted the source spectrum with background subtraction. We focused exclusively on order 33 $(2.29-2.33 \mu \mathrm{m})$ which samples CO $\nu=2-0$ transitions and telluric $\mathrm{CO}, \mathrm{H}_{2} \mathrm{O}$ and $\mathrm{CH}_{4}$ features. The optimally extracted spectra, which had signals-to-noise exceeding 100 on each night, were forward modeled as described in Section 3.

\subsection{High Angular Resolution Near-infrared Imaging}

WISE J0720-0846 was observed with the sodium Laser Guide Star Adaptive Optics system (van Dam et al. 2006; Wizinowich et al. 2006) and facility Near-infraRed Camera 2 (NIRC2) on the $10 \mathrm{~m}$ Keck II Telescope on 2014 January 19 (UT). Conditions were clear, dry and windy with slightly poor seeing $\left(\sim 1^{\prime \prime}\right)$. The narrow field of view (FOV) camera of NIRC2 was utilized, providing an image scale of $9.963 \pm$

\footnotetext{
11 Image reduction and analysis facility (Tody 1986).
} 


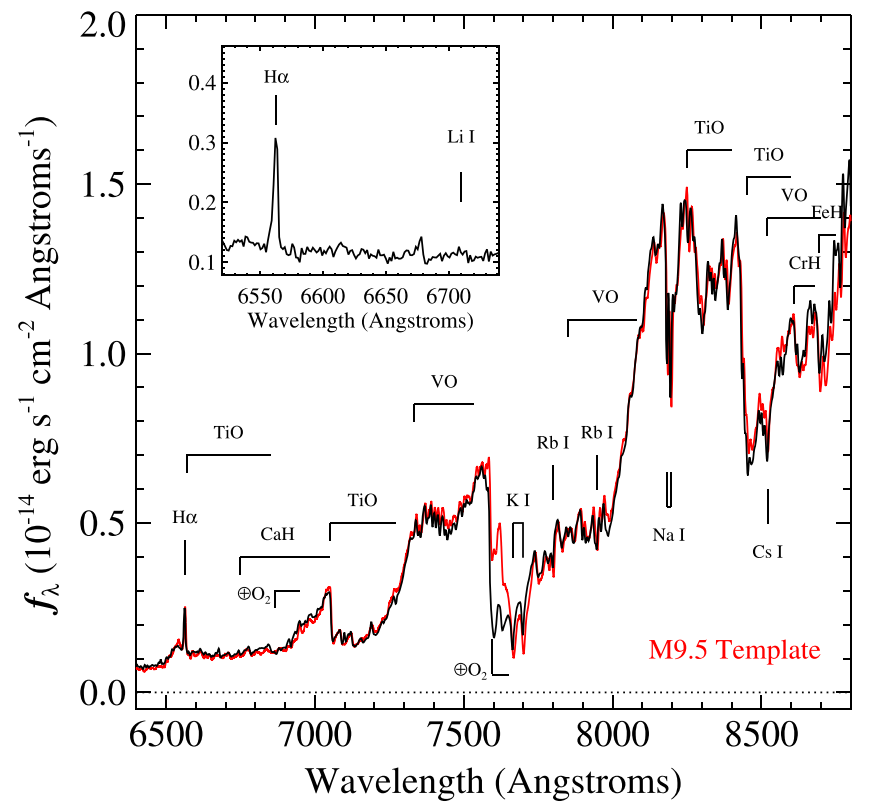

Figure 1. Red optical RCSpec spectrum of WISE J0720-0846 (black line) scaled to apparent flux units using $R_{J}=16.80$ from SuperCOSMOS (Hambly et al. 2001a, 2001b, 2001c). The spectrum is compared to an M9.5 spectral template created by merging M9 and L0 SDSS templates from Bochanski et al. (2007, red line) that is scaled to match WISE J0720-0846 in the 7400-7500 region. Both spectra are smoothed to a common resolution of $\lambda / \Delta \lambda=1200$. Primary atomic and molecular absorption features are labeled, as well as uncorrected telluric $(\oplus)$ bands in the WISE J0720-0846 spectrum. The inset box shows a close-up of the 6520-6730 ̊̊ region, revealing the presence of $\mathrm{H} \alpha$ emission and absence of $\mathrm{Li} \mathrm{I}$ absorption.

0.011 mas pixel $^{-1}$ (Pravdo et al. 2006) over a 10'.2 $\times 10^{\prime \prime} .2$ area. We used the $\mathrm{MKO}^{12} H$-band filter, and obtained six $60 \mathrm{~s}$ integrations using a three-position dither pattern with variable step size. While the LGS provided the wavefront reference source for AO correction, tip-tilt aberrations and slow variations were measured by monitoring the $R=16.8 \mathrm{mag}$ field star USNO 0812-0137390 located 20" northeast from WISE J0720-0846. As this tip-tilt star is faint, and conditions were marginal, we were only able to achieve a Strehl of $1.4 \%$ with these data.

Data were reduced using custom routines to perform flatfielding, background subtraction, bad-pixel correction, and shifting-and-stacking. The reduced image is shown in Figure 3. The poor strehl is evident in the broad wings of the pointspread function (PSF), which has a azimuthally averaged FWHM of 0.23 , and there is a slight elongation perpendicular to the direction of the tip-tilt star. These data are analyzed further in Section 4.

\subsection{Red Optical Photometric Monitoring}

WISE J0720-0846 was monitored for 13 non-consecutive days between 2013 December 30 and 2014 February 16 (UT) with the TRAnsiting Planets and PlanetesImals Small Telescope (TRAPPIST; Jehin et al. 2011), a $0.6 \mathrm{~m}$ robotic telescope located at La Silla Observatory in Chile. The telescope is equipped with a thermoelectrically-cooled $2 \mathrm{~K} \times 2 \mathrm{~K} \mathrm{CCD}$ camera with a 0.65 pixel scale and a $22^{\prime} \times 22^{\prime}$ FOV. Light is passed through a broad-band $I+z$ filter with $>90 \%$

\footnotetext{
$\overline{12}$ Mauna Kea Observatories near-infrared filter set (Simons \& Tokunaga 2002. Tokunaga et al. 2002).
}
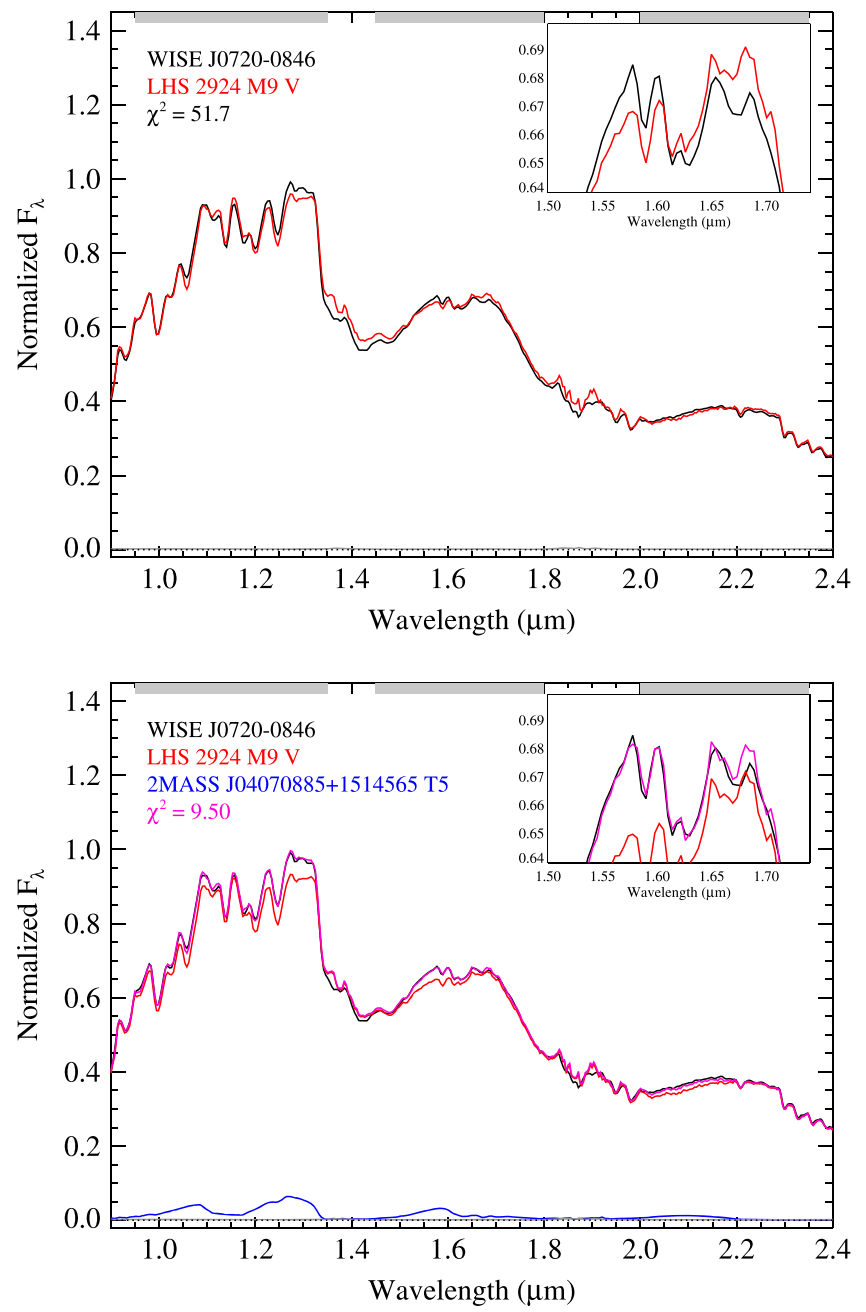

Figure 2. Top: normalized SpeX prism spectrum of WISE J0720-0846 (black line) compared to the best-fit single source fit from the SpeX Prism Library, the M9 LHS 2924 (red line, data from Burgasser \& McElwain 2006). The inset box focuses on the $1.62 \mu \mathrm{m}$ "dip" feature in the spectrum of WISE J07200846. Fit regions are indicated by the gray bars at top. Bottom: spectrum of WISE J0720-0846 compared to the best-fit binary template, a combination of LHS 2924 (red line) and the T5 2MASS J04070885+1514565 (blue line; data from Burgasser et al. 2004) relatively normalized according to the $M_{K}$ spectral type relation of Looper et al. (2007). The combined-light spectrum (purple line) coincides well with that of WISE J0720-0846, including replication of the dip feature and flux excesses at 1.3, 1.6 and $2.1 \mu \mathrm{m}$

transmission from $0.75-1.1 \mu \mathrm{m}$, the long-wavelength cutoff set by the quantum efficiency of the CCD detector. Individual exposures of $40 \mathrm{~s}$ each were obtained for continuous periods ranging from 4.8 to $8.5 \mathrm{hr}$, for a total of $73.6 \mathrm{hr}$ on source.

Data were reduced as described in Gillon et al. (2013). After a standard pre-reduction (bias, dark, flatfield correction), aperture photometry was performed using IRAF/DAOPHOT2 (Stetson 1987). Differential photometry was then determined by comparison to a grid of non-varying background stars, and the overall light curve was normalized to its mean value. Relative light curves for WISE J0720-0846 and a nearby comparison star 2MASS J07200688-0846504 $(J=12.71 \pm$ $\left.0.02, J-K_{s}=0.39 \pm 0.03\right)$ are shown in Figures 4 and 5 as a function of Modified Heliocentric Julian Day ${ }^{13}$ (HJD). These light curves are discussed in detail in Section 3.

\footnotetext{
13 Julian Day corrected for the Earth-Sun distance, minus 2450000.
} 
Table 1

Hamilton Spectrograph Observations of WISE J0720-0846

\begin{tabular}{|c|c|c|c|c|c|c|}
\hline UT Date & $\begin{array}{l}\text { MJD } \\
\text { (days) }\end{array}$ & $\mathrm{S} / \mathrm{N}^{\mathrm{a}}$ & $\begin{array}{c}V_{\text {rad }} \\
\left(\mathrm{km} \mathrm{s}^{-1}\right)\end{array}$ & $\begin{array}{c}V_{\text {rad }}(\mathrm{H} \alpha) \\
\left(\mathrm{km} \mathrm{s}^{-1}\right)\end{array}$ & $\begin{array}{c}\mathrm{H} \alpha \mathrm{EW} \\
(\AA)\end{array}$ & $\begin{array}{c}\log _{10} L_{H \alpha} / L_{\text {bol }} \\
(\operatorname{dex})\end{array}$ \\
\hline 2013 Dec 21 & 56648.02218 & 5 & $+82.6 \pm 0.5$ & $+76 \pm 5$ & $2 \pm 1$ & $-5.2 \pm 0.6$ \\
\hline 2013 Dec 22 & 56648.93341 & 15 & $+82.5 \pm 0.5$ & $+82 \pm 5$ & $1 \pm 1$ & $-5.9 \pm 1.3$ \\
\hline 2014 Jan 21 & 56678.92950 & 5 & $+82.1 \pm 0.5$ & $+79 \pm 5$ & $5 \pm 3$ & $-5.0 \pm 1.0$ \\
\hline 2014 Feb 23 & 56711.82038 & 25 & $+83.0 \pm 0.4$ & $+79 \pm 5$ & $13 \pm 5$ & $-4.3 \pm 0.4$ \\
\hline $2014 \mathrm{Feb} 24$ & 56712.80845 & 15 & $+82.9 \pm 0.4$ & $+80 \pm 5$ & $5 \pm 2$ & $-4.8 \pm 0.4$ \\
\hline
\end{tabular}

a Median signal-to-noise ratio in the 7000-8000 ̊̊ region.

Table 2

Radial and Rotational Velocities from NIRSPEC Observations

\begin{tabular}{lccl}
\hline \hline UT Date & MJD & $\begin{array}{c}\text { RV } \\
\left(\mathrm{km} \mathrm{s}^{-1}\right)\end{array}$ & $\begin{array}{l}v \sin i \\
\left(\mathrm{~km} \mathrm{~s}^{-1}\right)\end{array}$ \\
\hline 2014 Jan 19 & 56676.51 & $+84.3 \pm 0.4$ & $6.9 \pm 0.7$ \\
2014 Jan 20 & 56677.50 & $+83.2 \pm 0.4$ & $8.9 \pm 0.7$ \\
2014 Mar 10 & 56726.22 & $+84.3 \pm 0.7$ & $8.5 \pm 1.2$ \\
2014 Apr 12 & 56759.24 & $+83.6 \pm 0.3$ & $1.3 \pm 0.7$ \\
\hline
\end{tabular}

In addition, nightly astrometry measurements for WISE J0720-0846 were determined from the December through February data, as well as a short sequence of 23 frames obtained on 2014 September 16 (UT). To register the astrometric frames, we matched 150 bright, red sources to the PPMXL catalog (Roeser et al. 2010), using a third-order polynomial (with cross-terms) to map pixels to position. Observations on either side of meridian transit had root mean square deviations of order 15 mas; however, a larger shift of order 30-60 mas was found between frames taken before and after meridian. We therefore adopted the mean of pre- and post-meridian measurements as nightly values, and used the cross-meridian shift as an estimate of uncertainty. These values (equinox J2000) are listed in Table 3 and discussed further in Section 3.

\section{SYSTEM CHARACTERIZATION}

\subsection{Updated Astrometry}

With 13 nights of precision TRAPPIST astrometry we attempted to validate and improve the parallax and proper motion measurements reported by Scholz (2014). As our new measurements span less than a year, we combined the survey data reported by Scholz with our TRAPPIST measurements, rejecting only the SuperCosmos Sky Survey H $\alpha$ and Short-R survey measurements (Parker et al. 2005) for which the PSFs were reported to be elliptical. A parallax solution was determined using a Monte Carlo Markov Chain (MCMC) analysis with the Metropolis-Hastings sampling algorithm. Starting from the parameters determined by Scholz (parallax, proper motion) and the average position of the target, we performed a $10^{5}$-step random walk, at each step varying the astrometric parameters using normal distributions. We compared the $F$-distribution probability distribution function for $\chi^{2}$ residual values computed before and after each step to a uniform distribution as our acceptance ratio. Ignoring the first $10 \%$ of the chain, we calculated the mean and standard deviation of the parameters in the remaining steps, weighting each solution by the $F$-distribution. Our results, displayed in Figures 6 and 7 and listed in Table 5, are in agreement with Scholz (2014) but with improved uncertainties, particularly in proper motion. Our derived parallax distance, $6.0 \pm 1.0 \mathrm{pc}$, is more in line with the spectrophotometric estimates reported by
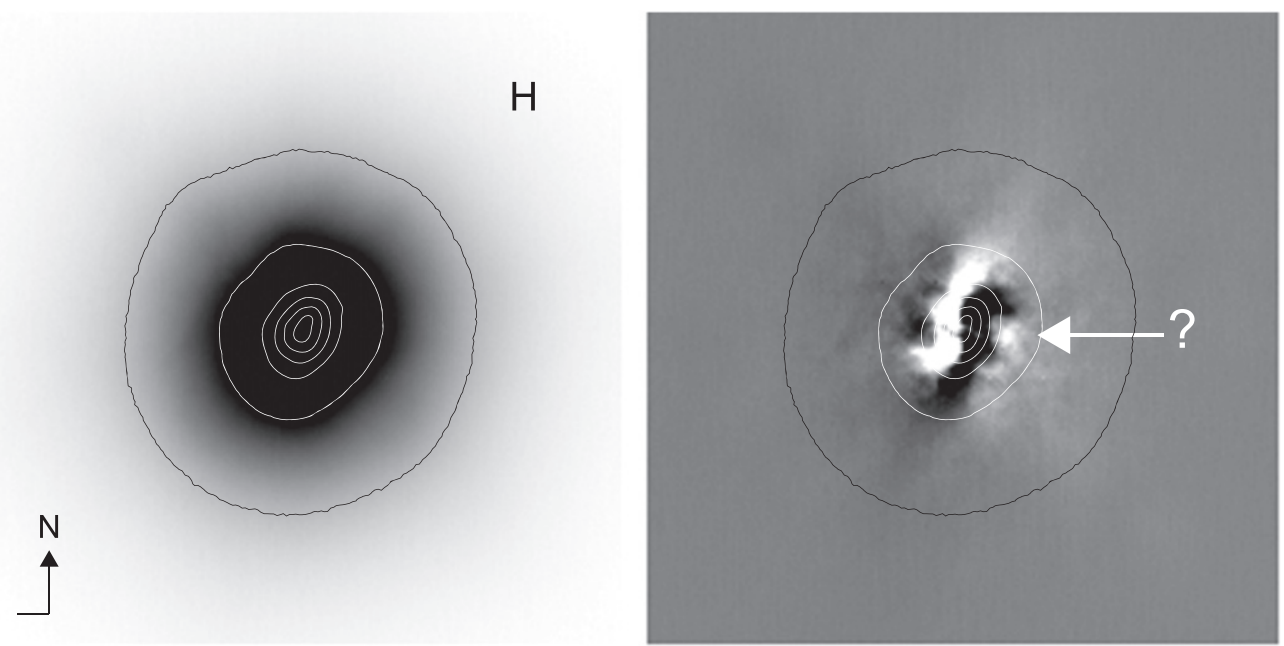

Figure 3. Left: $H$-band image of WISE J0720-0846 taken with NIRC2 LGSAO. Image is $2^{\prime \prime}$ on a side with north up and east to the left. White contours indicate 0.1 , $0.3,0.5,0.7$, and 0.9 times the peak flux of the source; black contours indicate the flux level of the predicted companion. Right: same image but self-subtracted after $180^{\circ}$ rotation. A candidate faint source (arrow) is seen west of the primary at a separation of 0.14 (1.0 AU projected separation). 

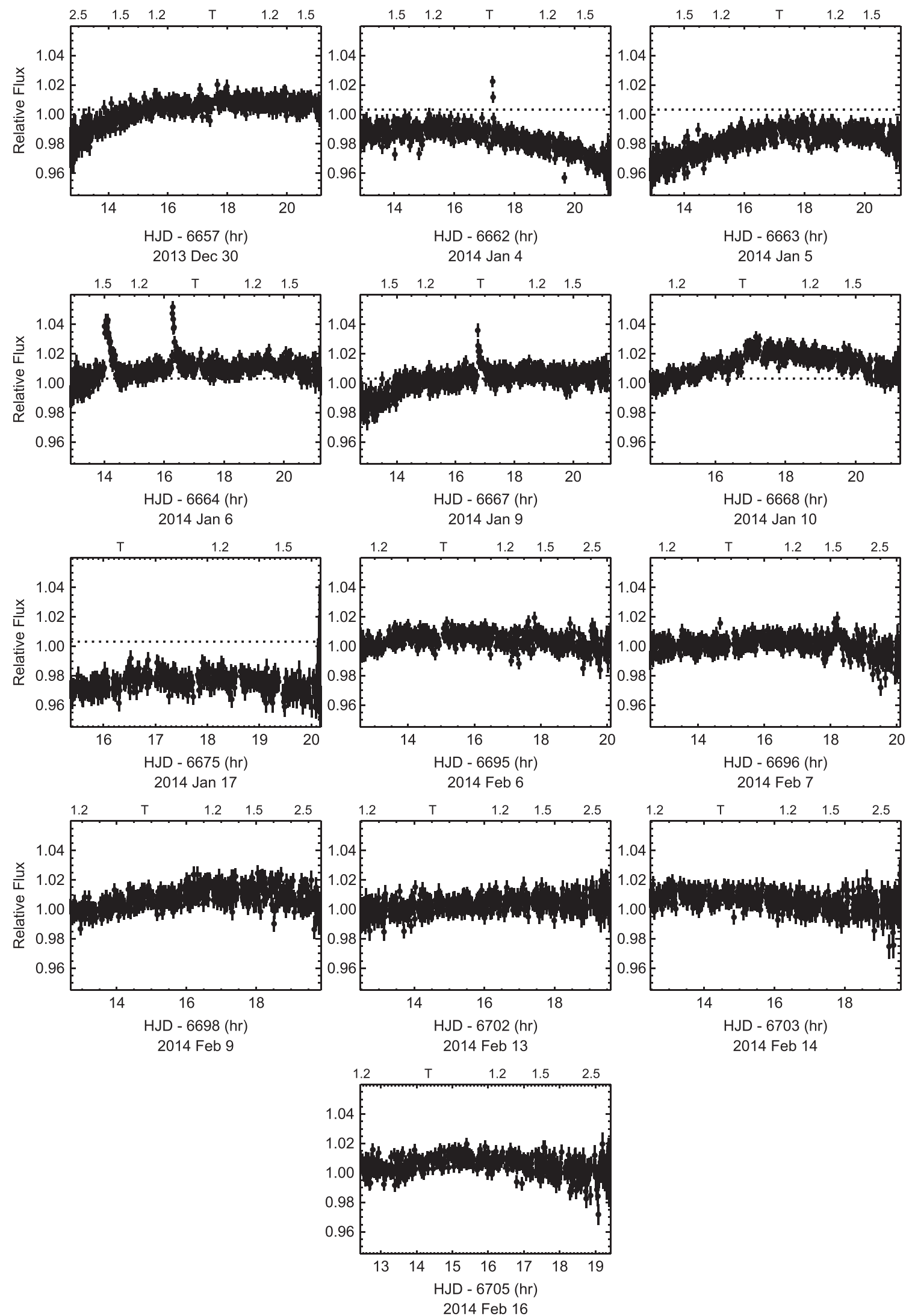

Figure 4. TRAPPIST relative light-curves for WISE J0720-0846 for each of the 13 nights this source was observed between 2013 December 30 and 2014 February 16 (UT). Flux values have been normalized to a global mean. Above each panel the airmasses are indicated, as well as the time of transit $(z=1.067)$. No correction for airmass-dependent brightness changes has been made in this plot. 

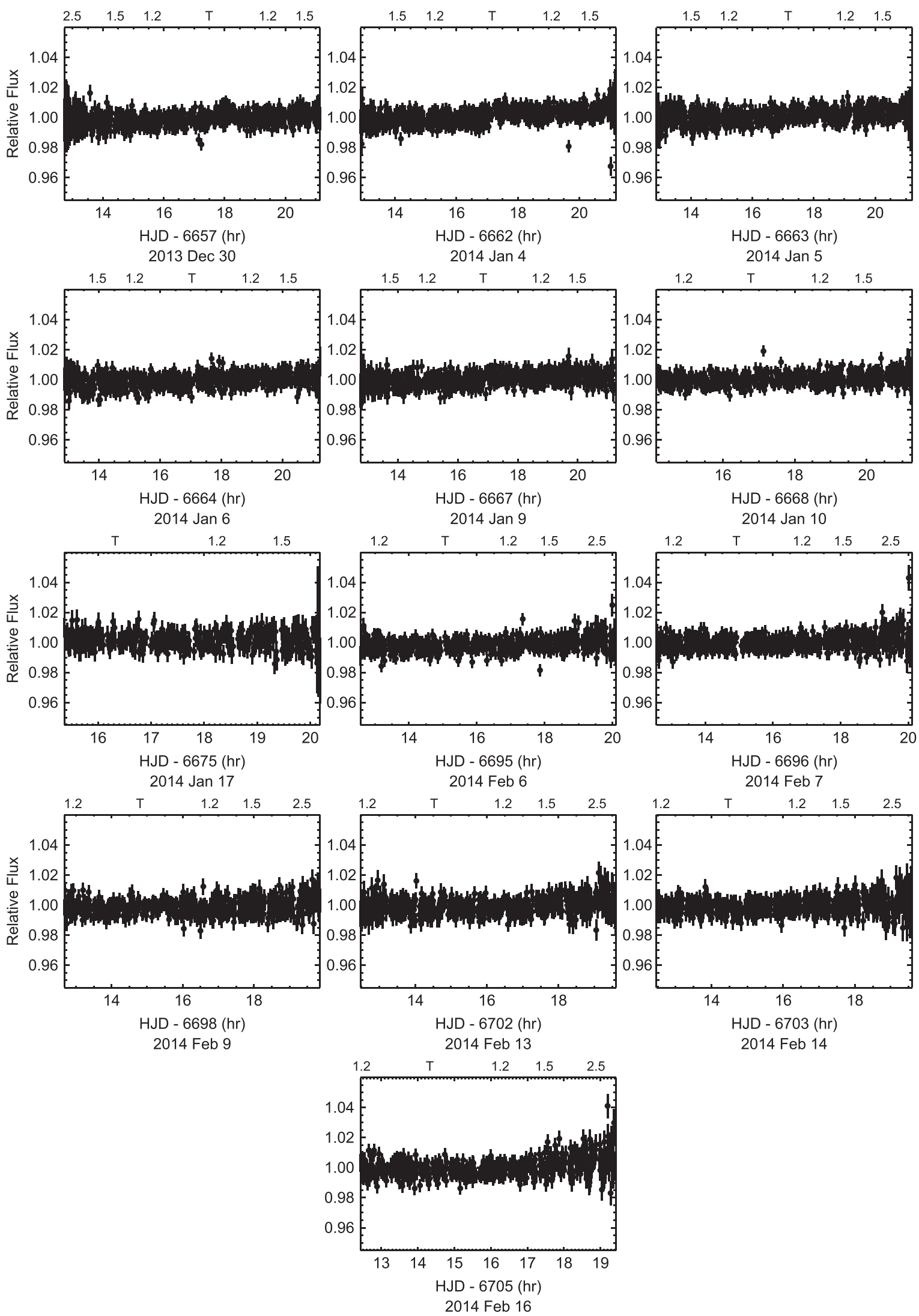

Figure 5. Same as Figure 4, but for the nearby comparison star 2MASS J07200688-0846504, 0.06 mag fainter in the TRAPPIST bandpass. 
Table 3

TRAPPIST Astrometry

\begin{tabular}{|c|c|c|c|c|c|c|}
\hline UT Date & MJD & $\begin{array}{c}\alpha \\
(\mathrm{J} 2000)\end{array}$ & $\begin{array}{c}\sigma_{\alpha} \\
(\mathrm{mas})\end{array}$ & $\begin{array}{c}\delta \\
(\mathrm{J} 2000)\end{array}$ & $\begin{array}{c}\sigma_{\delta} \\
(\mathrm{mas})\end{array}$ & $\overline{\text { \# Frames }}$ \\
\hline 2014 Jan 4 & 56662.5 & $07^{\mathrm{h}} 20^{\mathrm{m}} 03^{\mathrm{s}} 2108$ & 31 & $-08^{\circ} 46^{\prime} 51^{\prime} 830$ & 28 & 573 \\
\hline 2014 Jan 5 & 56663.5 & $07^{\mathrm{h}} 20^{\mathrm{m}} 03^{\mathrm{s}} .2108$ & 14 & $-08^{\circ} 46^{\prime} 51^{\prime} 832$ & 34 & 575 \\
\hline 2014 Jan 9 & 56667.5 & $07^{\mathrm{h}} 20^{\mathrm{m}} 03^{\mathrm{s}} \cdot 2100$ & 17 & $-08^{\circ} 46^{\prime} 51^{\prime} 832$ & 33 & 586 \\
\hline 2014 Jan 10 & 56668.5 & $07^{\mathrm{h}} 20^{\mathrm{m}} 03^{\mathrm{s}} \cdot 2102$ & 25 & $-08^{\circ} 46^{\prime} 51^{\prime} 825$ & 36 & 492 \\
\hline 2014 Jan 17 & 56675.5 & $07^{\mathrm{h}} 20^{\mathrm{m}} 03^{\mathrm{s}} .2091$ & 16 & $-08^{\circ} 46^{\prime} 51^{\prime} 827$ & 43 & 325 \\
\hline 2014 Feb 9 & 56698.5 & $07^{\mathrm{h}} 20^{\mathrm{m}} 03^{\mathrm{s}} .2058$ & 41 & $-08^{\circ} 46^{\prime} 51^{\prime} 815$ & 28 & 486 \\
\hline 2014 Feb 13 & 56702.5 & $07^{\mathrm{h}} 20^{\mathrm{m}} 03^{\mathrm{s}} .2052$ & 29 & $-08^{\circ} 46^{\prime} 51^{\prime} 809$ & 29 & 483 \\
\hline 2014 Feb 14 & 56703.5 & $07^{\mathrm{h}} 20^{\mathrm{m}} 03^{\mathrm{s}} .2063$ & 48 & $-08^{\circ} 46^{\prime} 51^{\prime} 822$ & 57 & 480 \\
\hline 2014 Feb 16 & 56705.5 & $07^{\mathrm{h}} 20^{\mathrm{m}} 03^{\mathrm{s}} \cdot 2045$ & 34 & $-08^{\circ} 46^{\prime} 51^{\prime} 809$ & 29 & 472 \\
\hline 2014 Sep 17 & 56908.9 & $07^{\mathrm{h}} 20^{\mathrm{m}} 03^{\mathrm{s}} .2214$ & 16 & $-08^{\circ} 46^{\prime} 51^{\prime \prime} 750$ & 16 & 23 \\
\hline
\end{tabular}
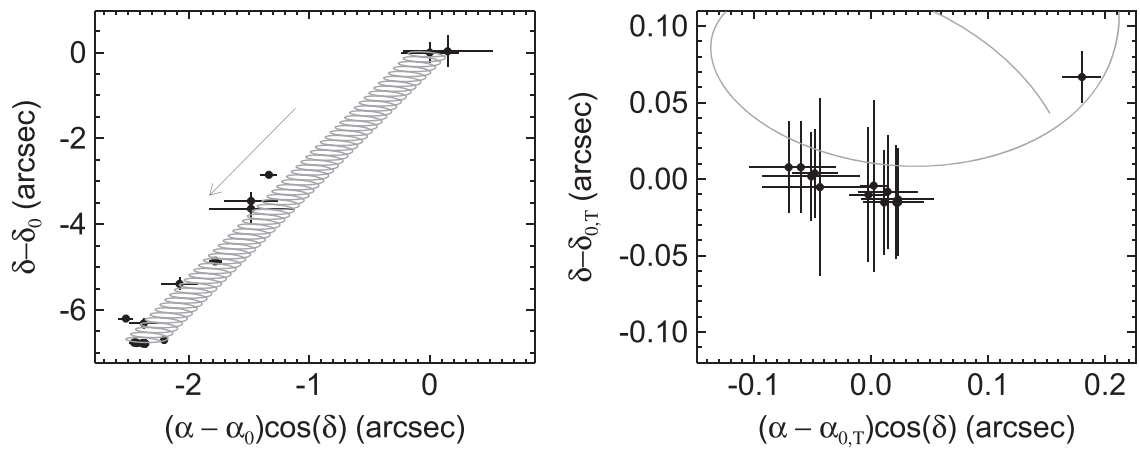

Figure 6. (Left): astrometric solution for WISE J0720-0846 (gray line) compared to positional data compiled by Scholz (2014) and reported here. Positions are shown as offsets from a zero point position of $07^{\mathrm{h}} 20^{\mathrm{m}} 03^{\mathrm{s}} 47-08^{\circ} 46^{\prime} 36^{\prime \prime} 26$ at MJD $=35429.0$ (1955 November 17 UT) The arrow indicates the general direction of proper motion. (Right): close-up view of TRAPPIST astrometry.

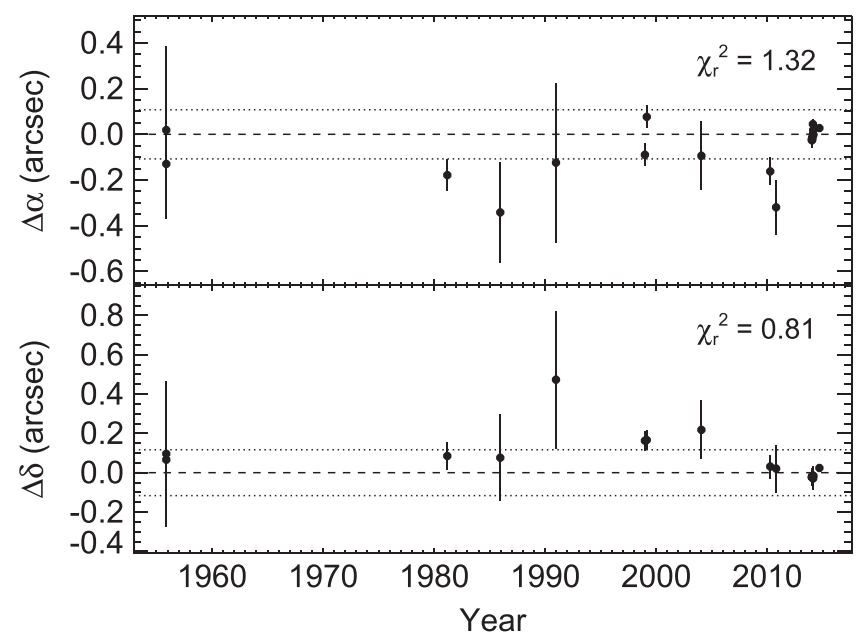

Figure 7. Residuals between position measurements and derived astrometric solution in R.A. (top) and decl. (bottom). Standard deviations in the residuals are 108 mas and 117 mas, respectively (dotted lines), and reduced $\chi^{2}$ values are indicated.

Scholz, and the improvement is driven largely by the more accurate TRAPPIST astrometry. We aim to continue monitoring this source for further improvement.

\subsection{Spectral Classification}

The reduced RC Spec spectrum of WISE J0720-0846 is compared to an M9.5 spectral template in Figure 1, produced
Table 4

Optical Classification Indices and Spectral Types for WISE J0720-0846

\begin{tabular}{|c|c|c|}
\hline Index & Value & SpT \\
\hline \multicolumn{3}{|c|}{ Reid et al. (1995) } \\
\hline TiO5 & $0.463 \pm 0.007$ & $\ldots$ \\
\hline $\mathrm{CaH} 1$ & $0.927 \pm 0.020$ & $\cdots$ \\
\hline $\mathrm{CaH} 2$ & $0.454 \pm 0.006$ & $\cdots$ \\
\hline $\mathrm{CaH} 3$ & $0.736 \pm 0.010$ & $\ldots$ \\
\hline \multicolumn{3}{|c|}{ Kirkpatrick et al. (1999) } \\
\hline $\mathrm{CrH}-\mathrm{a}$ & $1.091 \pm 0.009$ & M9/L0 \\
\hline Rb-b/TiO-b & $0.635 \pm 0.009$ & M9/L0 \\
\hline Cs-a/VO-b & $0.768 \pm 0.008$ & M9/L0 \\
\hline \multicolumn{3}{|c|}{ Lépine et al. (2003) } \\
\hline VO1 & $0.737 \pm 0.006$ & M9.7 \\
\hline $\mathrm{VO} 2$ & $0.364 \pm 0.003$ & M8.6 \\
\hline TiO6 & $1.858 \pm 0.017$ & $\ldots$ \\
\hline $\mathrm{TiO} 7$ & $0.521 \pm 0.004$ & M8.0 \\
\hline Color-M & $9.93 \pm 0.07$ & M9.1 \\
\hline \multicolumn{3}{|c|}{ Lépine et al. (2007) } \\
\hline$\zeta$ & $1.034 \pm 0.018$ & $\mathrm{dM}$ \\
\hline
\end{tabular}

by merging the M9 and L0 SDSS templates from Bochanski et al. (2007). This hybrid template provides the best overall match to the optical spectral shape of WISE J0720-0846. 
Wavelength $(\mu \mathrm{m})$

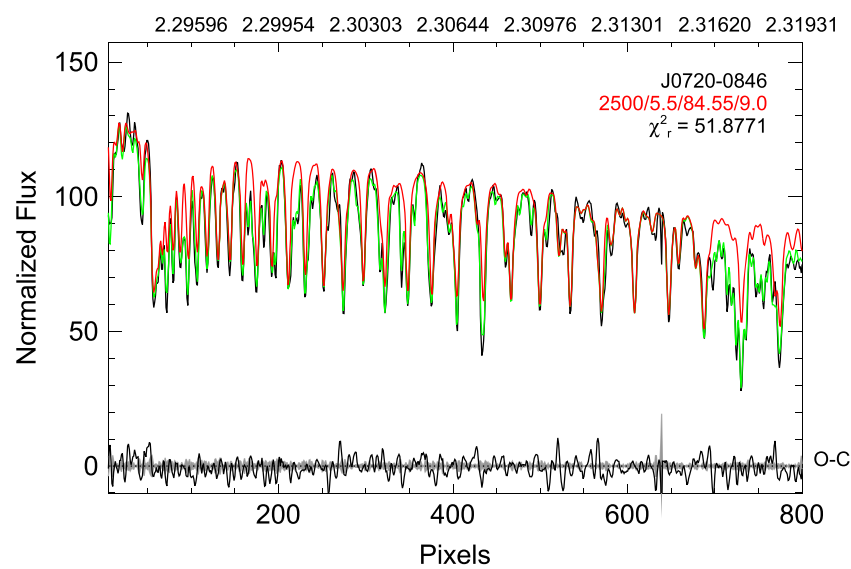

Figure 8. High-resolution $(\lambda / \Delta \lambda=20,000) K$-band spectrum of WISE J0720 0846AB (black line) from 2014 March 10 (UT) compared to a best-fit model combining dwarf spectrum (red line) and telluric absorption (green line) components. The difference between data and model $(\mathrm{O}-\mathrm{C})$ is shown in black at the bottom of the plot and is dominated by fringing residuals; uncertainty spectrum is indicated in gray. The forest of $\mathrm{CO}$ bandheads allow us to precisely pin down the radial and rotational velocity of the primary of this system.

Spectral indices from Kirkpatrick et al. (1999), Lépine et al. $(2003,2007)$ are also consistent with this classification and indicate solar metallicity (Table 4). We detect no $6708 \AA \mathrm{Li}$ I absorption in these data or the co-added high resolution spectrum, to a $3 \sigma$ equivalent width limit of $E W<0.15 \AA$; we also find no indication of low surface gravity in the strengths of alkali lines or TiO/VO bands (Martn et al. 1999; Cruz et al. 2009). Both observations imply a mass $\gtrsim 0.06 M_{\odot}$ and age $\gtrsim 100$ Myr for this source (Bildsten et al. 1997; Kirkpatrick et al. 2008). $\mathrm{H} \alpha$ emission is detected, as described below.

For our near-infrared data, we compared the SpeX spectrum to 727 equivalent spectra of optically-classified M and L dwarfs drawn from the SpeX Prism Library (SPL; Burgasser 2014). Following the fitting methodology described in Burgasser et al. (2010), we found a best match to the M9 dwarf LHS 2924 (Probst \& Liebert 1983; Figure 2) and a $F$-distribution weighted mean to all templates of M9.0 \pm 0.7 . Subtle variances between the spectrum of WISE J0720-0846 and LHS 2924 are discussed in detail below. These results confirm the near-infrared classification derived by Kirkpatrick et al. (2014) and establish congruence between the optical and nearinfrared spectral morphologies.

\subsection{Kinematics}

The small proper motion of WISE J0720-0846, $121.7 \pm 0.3$ mas $\mathrm{yr}^{-1}$, translates into a relatively low tangential velocity, 3.5 $\pm 0.6 \mathrm{~km} \mathrm{~s}^{-1}$. We determined the radial velocity of this source from our high-resolution optical and near-infrared data. For the optical data, we cross-correlated each of the spectra across several orders in the 6600-9000 $\AA$ range to the RV standard Gl $251\left(V_{r}=22.91 \pm 0.10 \mathrm{~km} \mathrm{~s}^{-1}\right.$; Nidever et al. 2002) to determine a relative offset. The widths of the cross-correlation peak in each order yield velocity uncertainties of $0.5-1.0$ $\mathrm{km} \mathrm{s}^{-1}(\sim 1 / 5$ of the resolution), which average down to $0.3-0.5 \mathrm{~km} \mathrm{~s}^{-1}$ after combining all orders. The NIRSPEC data were forward-modeled using a custom MCMC implementation of the method described in Blake et al. (2010). The Solar atlas of Livingston \& Wallace (1991) was used to model telluric absorption and the BT-Settl atmosphere models (Allard et al. 2012) were used to model the spectrum of WISE J0720-0846. Spectral models with $T_{\text {eff }}=2300-2500 \mathrm{~K}$ and $\log g=5.5$ (cgs) provided the best fits. Figure 8 shows that this procedure provides an accurate fit to the data, with residuals dominated by uncorrected fringing. Radial velocity and rotational broadening were among the parameters modeled, and the mean and standard deviation of their marginalized distributions in the MCMC chain were used as estimates of their measured values and uncertainties. For the radial velocities, uncertainties range over $0.3-0.7 \mathrm{~km} \mathrm{~s}^{-1}$. Tables 1 and 2 list all radial velocity measurements over the 11 epochs observed.

Both data sets are consistent with a large radial motion for WISE J0720-0846, with averages of $+82.5 \pm 0.4 \mathrm{~km} \mathrm{~s}^{-1}$ from the Hamilton data and $+83.7 \pm 0.4 \mathrm{~km} \mathrm{~s}^{-1}$ from the NIRSPEC data $^{14}$, in clear contrast to its small tangential motion. The total spatial velocity vector in the Local Standard of $\operatorname{Rest}^{15}$ is $(U, V$, $W)=(-47.5,-47.6,8.0) \pm(0.4,0.4,0.5) \mathrm{km} \mathrm{s}^{-1}$, which falls well outside the $1 \sigma$ distribution of local late-type $\mathbf{M}$ dwarfs, $\left(\sigma_{U}, \sigma_{V}, \sigma_{W}\right)=(32,20,17) \mathrm{km} \mathrm{s}^{-1}$ (Reid et al. 2002; Reiners \& Basri 2009). Its Galactic orbit, computed as described in Burgasser et al. (2009), is modestly eccentric, spanning Galactic radii of 4-9 kpc (assuming a Solar radius of $8.5 \mathrm{kpc}$ ). Using the criteria of Leggett (1992), we assign this source to the old disk kinematic population, suggesting a "mature" age of 0.5-5 Gyr (Eggen 1969).

\subsection{Magnetic Activity}

$\mathrm{H} \alpha$ emission is detected in both the RC Spec and Hamilton Spectrograph data. In the low-resolution data, we measure an equivalent width $E W=-5.2 \pm 0.3 \AA$. Using a $\log _{10} \chi \equiv \log _{10} f_{6560} / f_{\text {bol }}=-5.4 \pm 0.1$ from Walkowicz et al. (2004), we determine $\log _{10} L_{H \alpha} / L_{\text {bol }}=\log _{10}(\chi E W)=-4.68 \pm$ 0.06, which is typical for M9 dwarfs in the vicinity of the Sun (Schmidt et al. 2007; West et al. 2011).

Figure 9 displays our Hamilton spectral data of WISE J07200846 around the $6563 \AA \mathrm{H} \alpha$ line. Continuum emission is weakly detected at red wavelengths in these data, but the $\mathrm{H} \alpha$ line is unambiguous, with both broad and highly variable emission. Equivalent widths (absolute values) range from $\lesssim 1$ to $13 \pm 5 \AA$, corresponding to $\log _{10} L_{H \alpha} / L_{\text {bol }} \lesssim-6$ (inactive) to -4.3 ( $>2 \times$ "normal" activity). These lines are also wellresolved, with FWHM of $\sim 40-60 \mathrm{~km} \mathrm{~s}^{-1}$, over five times the NIRSPEC-derived rotational velocity (see below). The $\mathrm{H} \alpha$ line centers are nevertheless aligned with absorption line Doppler shifts to within $5 \mathrm{~km} \mathrm{~s}^{-1}$, ruling out jets or accretion projected along the line of sight.

In addition to emission line variability, the TRAPPIST light curve shows several impulsive bursts on HJD 6662, 6664 and 6667, visible in Figure 4 and shown in detail in Figure 10. These have relatively low power, with peak fluxes $4-8 \%$ above the local continuum. Three exhibit classic flare profiles, with impulsive rises $(<1$ minutes $)$ followed by exponential declines (2-5 minutes). In contrast, the burst on HJD 6664.5859, one of

\footnotetext{
${ }^{14}$ We attribute the marginal difference $(2 \sigma)$ between these mean values to a systematic shift between the instruments; see Ford (2005).

${ }^{15}$ Assuming a right-handed Cartesian coordinate system centered on the Sun with $U$ pointed radially away from the Galactic center, $V$ in the direction of Galactic rotation, and $W$ toward the Galactic north pole. We also adopt a Local Standard of Rest motion of $(U, V, W)_{\mathrm{LSR}}=(11.1,12.24,7.25) \pm(0.7,0.5,0.4)$ $\mathrm{km} \mathrm{s}^{-1}$ (Schönrich et al. 2010).
} 


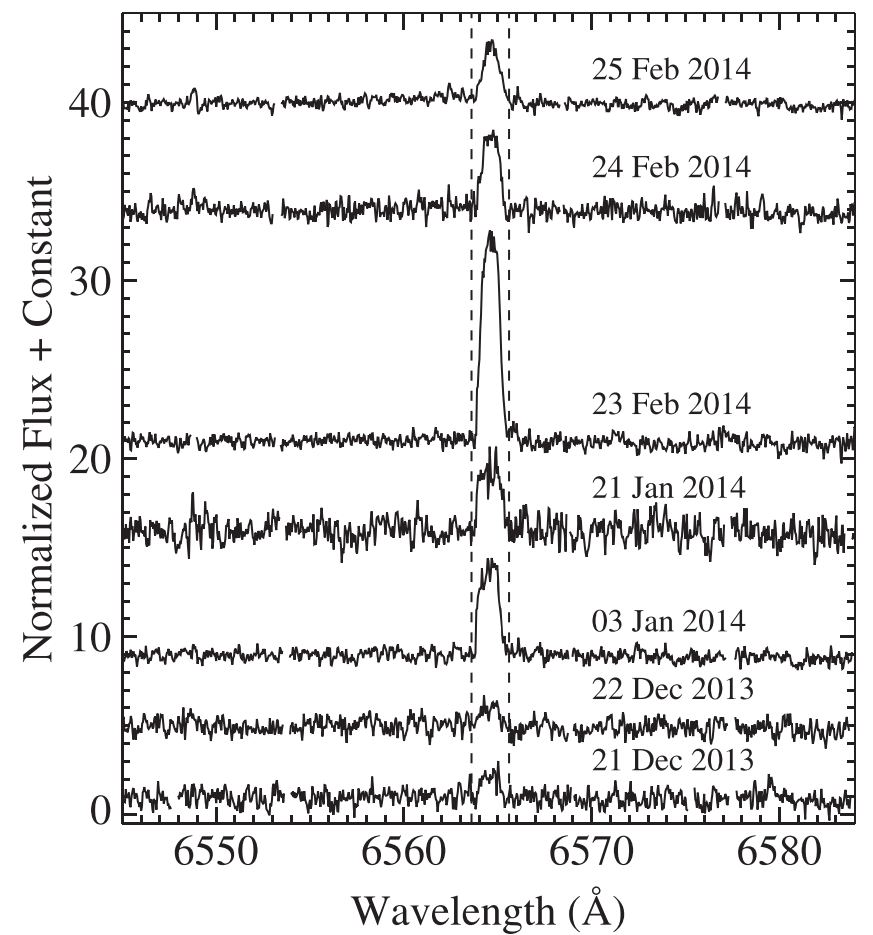

Figure 9. Lick Hamilton spectra of WISE J0720-0846 showing the $\mathrm{H} \alpha$ emission feature. All spectra are normalized such that the median continuum around the $\mathrm{H} \alpha$ line is unity; the spectra are then offset vertically for clarity. Variability in the amplitude of the $\mathrm{H} \alpha$ emission is clear. The dashed vertical lines encompass a region with velocity width of $\approx 90 \mathrm{~km} \mathrm{~s}^{-1}$. Wavelengths in this figure are heliocentric and plotted in air. Some residual sky lines have been removed manually from the spectra.
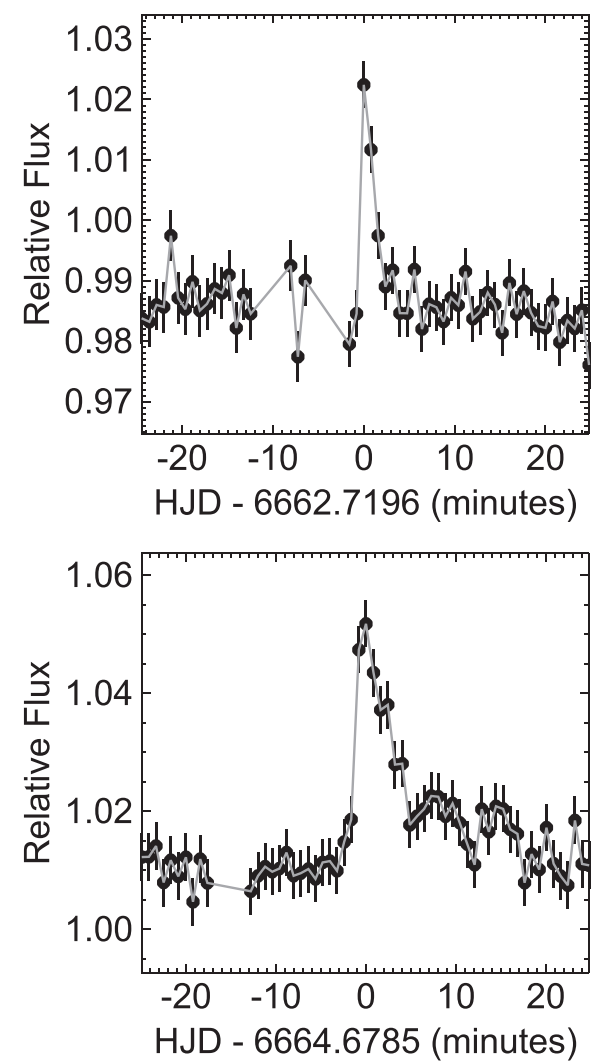

two in a 2 hr period, shows a more complex temporal structure, with a broad peak that persists for over 5 minutes followed by a 15 minutes decline with possible secondary bursts after 10 minutes. This may reflect an associated chain of flare events over an extended region, or a massive flare rotating out of view. These events indicate a $0.8 \%$ duty cycle of flare emission at the level of $2 \%$ above quiescence, somewhat below the typical duty cycles of late $\mathrm{M}$ dwarfs ( $3 \pm 1 \%$; Hilton et al. 2010).

Hence, while WISE J0720-0846 exhibits numerous signs of activity, including an unusually broad $\mathrm{H} \alpha$ line, the strength of its persistent emission and frequency of flaring are at or below the average of equivalently classified dwarfs. This is consistent with its old kinematic age and slow rotation rate (see below), although it should be noted that age-activity and rotationactivity trends are not well established in the late $\mathrm{M}$ and $\mathrm{L}$ dwarf regime (Gizis et al. 2000; Mohanty \& Basri 2003; Reiners \& Basri 2008; McLean et al. 2012).

\subsection{Rotation}

In addition to radial motion, our NIRSPEC analysis provides multi-epoch measurements of rotational broadening. We measure a consistent $v \sin i=8.0 \pm 0.5 \mathrm{~km} \mathrm{~s}^{-1}$, which is on the low end of, but consistent with, the rotational velocities of equivalently classified dwarfs (Reiners \& Basri 2008; Blake et al. 2010; McLean et al. 2012). Assuming a radius of $0.1 R_{\odot}$ (Burrows et al. 2001), this velocity corresponds to a maximum rotation period of $15 \pm 1 \mathrm{hr}$, consistent with period measurements for equivalent-mass objects (Irwin et al. 2011). Despite being on the low end of the $v \sin i$ distribution of late-
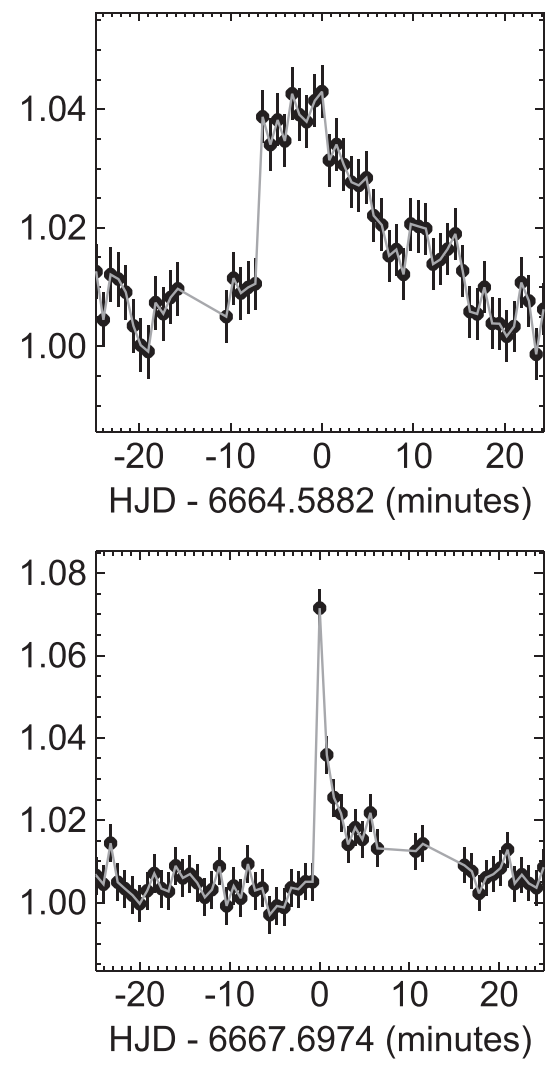

Figure 10. Individual broadband flares detected in the TRAPPIST light curve of WISE J0720-0846. Note the variation in vertical scale in each panel. Three of the bursts show a classical burst profile, with an impulsive rise and exponential decay. The burst at HJD 6664.59, which occurs $2.2 \mathrm{hr}$ before the burst at HJD 6664.68, is more extended in time, and may represent sequential flares or an extended, active spot. 

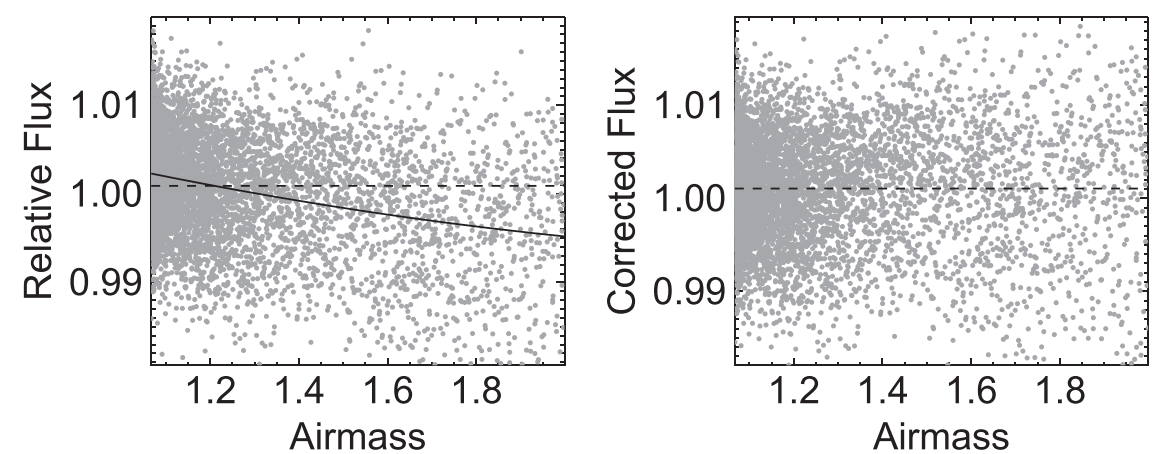

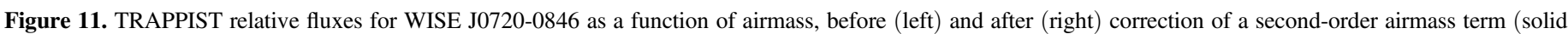

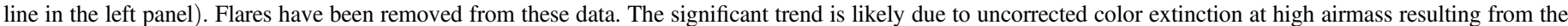
very different spectral energy distribution of WISE J0720-0846 compared to nearby comparison stars.

type $\mathrm{M}$ dwarfs, this source is still a rapid rotator, a likely explanation for its magnetic activity.

\subsection{Search for Photometric Variability}

To confirm the slow rotation of WISE J0720-0846, we searched for rotational modulation of surface features in the TRAPPIST light curve. As shown in Figure 4, the red optical brightness of this source exhibits considerable night-to-night variation, particularly prior to HJD 6695, the same period we detect flaring bursts. From HJD 6695 onward, WISE J0720-0846 is far more stable. Hereafter, we refer to these periods as the "active" and "quiescent" phases. For comparison, the relative fluxes of the nearby comparison star (0.06 mag fainter in the TRAPPIST bandpass) are steady throughout the observing period (Figure 5).

The nightly variations appear to be episodic, and we could find no clear period associated with them. We therefore normalized each night's light curve to search for persistent lowlevel variability. There is additional structure in the normalized light curves that arises from differential color extinction at large airmass (Bailer-Jones \& Lamm 2003). WISE J0720-0846 has a distinct spectral energy distribution in the $0.75-1$ $\mu \mathrm{m}$ TRAPPIST band compared to its neighboring sources, so color-dependent extinction is not corrected in relative photometry. Figure 11 shows source flux as a function of airmass. A statistically significant trend is present, reaching a deviation of $0.5 \%$ at an airmass of 2.0. In contrast, no significant trend is found in the comparison source. This airmass effect was corrected in the light curve of WISE J0720-0846 by dividing by a second order polynomial fit to airmass; we also rejected all measurements at airmasses greater than 2.0 to minimize residual bias. This reduced our total number of samples to 5895. We found no significant trends with airmass or seeing after the airmass correction was applied.

The corrected and nightly-normalized light curve was analyzed using the Phase Dispersion Minimization (PDM) technique (Stellingwerf 1978; Davies 1990). Our implementation of this method is described in Appendix A. We examined periods between 4 and $16 \mathrm{hr}$, the lower limit consistent with the most rapidly rotating brown dwarfs and the upper limit chosen to be just above our rotational velocity limit. Figures 12 and 13 display distributions of the PDM statistic $\Theta$ as a function of period for WISE J0720-0846 and the comparison star, respectively. The PDM of WISE J0720-0846 shows considerable structure, but none of the features exceed our significance threshold of $90 \%$. The phased light curve of the strongest period at $14.00 \pm 0.05 \mathrm{hr}$ displays a compelling, double-peaked pattern with an amplitude of $1.3 \pm 0.5 \%(2.6 \sigma)$. This period is consistent with a rotation axis inclination angle of $71^{\circ} \pm 8^{\circ}$. However, the $\chi^{2}$ of the phased photometric residuals relative to the smoothed light curve is formally consistent with a light curve without phasing. Several other statistics were investigated, including Lomb-Scargle analysis (Lomb 1976; Scargle 1982), and trial sinusoidal fits to the data were also insufficient for identifying a robust period. We therefore set a $3 \sigma$ upper limit of $1.5 \%$ on periodic variability in this source, although larger episodic variations are clearly present. Note that the comparison source also exhibits no significant period.

\section{THE BINARY NATURE OF WISE J0720-0846}

\subsection{Identification as a Spectral Binary}

As described above, the SpeX spectrum of WISE J0720-0846 is best matched to that of the M9 spectral standard LHS 2924. However, close inspection reveals specific discrepancies near 1.3 and $1.6 \mu \mathrm{m}$, and a subtle "dip" feature at $1.62 \mu \mathrm{m}$. Such features have been previously noted in the combined-light spectra of very low mass spectral binary systems with late-type $\mathrm{M}$ or $\mathrm{L}$ dwarf primaries and T dwarf secondaries (e.g., Cruz et al. 2004; Burgasser 2007b; Burgasser et al. 2010, 2011, 2012; Gelino \& Burgasser 2010; Kirkpatrick et al. 2012; Day-Jones et al. 2013; Deacon et al. 2014; Bardalez Gagliuffi et al. 2014). To assess whether WISE J0720-0846 is such a system, we used the fitting method described in Burgasser et al. (2010), comparing the spectrum of WISE J0720-0846 to 699 single star templates and 107,646 binary templates from the SPL. The latter were constructed using spectra of M7-L4 dwarfs for the primary and L9-T7 dwarfs for the secondary, with component spectra scaled to absolute magnitudes using the $M_{K}$ /spectral type relation of Looper et al. (2007).

Figure 2 displays the best fitting binary template, which consists of LHS 2924 paired to the T5 2MASS J04070885 +1514565 (Burgasser et al. 2004). This combination reproduces the excess flux at 1.3, 1.6, and $2.1 \mu \mathrm{m}$, as well as the shape of the $1.62 \mu \mathrm{m}$ dip feature, and is a statistically significant better match to the data based on an F-test comparison ( $>99 \%$ confidence). Marginalizing over all fits weighted by the $F$-distribution, we infer component types of M9 \pm 0.5 and $\mathrm{T} 5 \pm 0.7$, and $\Delta H=4.1 \pm 0.4$, implying that the secondary contributes only $2 \%$ of the combined light in this band. This system is similar in composition to the previously confirmed M8.5 + T5 spectral binaries 2MASS J032028390446358 (hereafter 2MASS J0320-0446; Blake et al. 2008; 

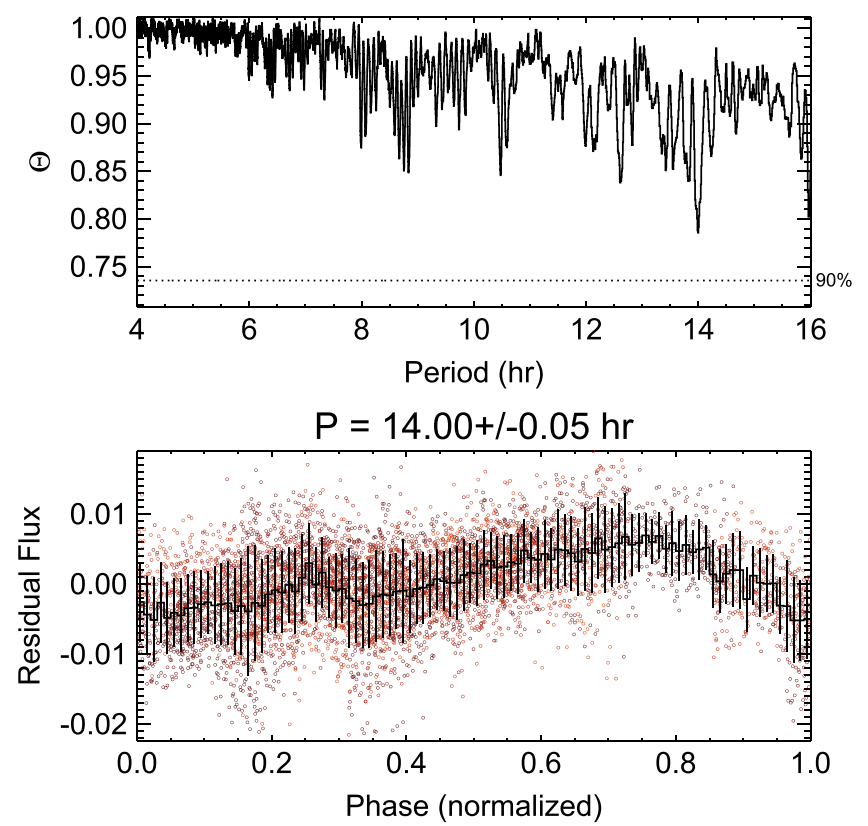

Figure 12. (Top): phased dispersion minimization statistic $\Theta$ as a function of period for the flare-cleaned, airmass-corrected, and daily normalized TRAPPIST light curve of WISE J0720-0846. A significance threshold of $90 \%$ based on Schwarzenberg-Czerny (1997) is indicated. None of the features exceed this threshold. (Bottom): phased light curve for the strongest candidate period at $14.00 \pm 0.05 \mathrm{hr}$. Individual measurements are indicated by small circles, where different shades of red indicate different cycles (17 in total). The black histogram with error bars (scatter per phase bin) delineates the mean phased light curve.


Figure 13. Same as Figure 12 but for the nearby comparison star 2MASS J07200688-0846504. The minima in $\Theta$ reflect the sampling window of the measurement (12 hr and harmonics) and are not significant.

Burgasser et al. 2008) and SDSS J000649.16-085246.3 (hereafter SDSS J0006-0852; Burgasser et al. 2012).

\subsection{Possible Detection of a Resolved Companion}

We searched for this potential companion using our NIRC2 $H$-band images. Given the somewhat poor observing conditions, the wings of the PSF reach $2 \%$ relative brightness at a relatively large radius of 0.6 (4.2 AU; Figure 3$)$. Only one point source is seen at larger radii, $4^{\prime \prime} 85$ to the southwest of WISE J0720-0846 with $\Delta H \approx 6$. However, a stationary optical and near-infrared counterpart to this source is seen in both Digital Sky Survey and 2MASS images, the former going back to 1955 , so it is likely an unrelated background star.

To probe tighter separations, we self-subtracted the combined image after rotating by $180^{\circ}$ and offsetting to minimize the total squared deviation, effectively using the source PSF as its own model. Figure 3 shows that a faint source emerges upon this subtraction, $139 \pm 14$ mas from the PSF center at a position angle of $262^{\circ} \pm 2^{\circ}$, corresponding to a projected separation of $0.84 \pm 0.17 \mathrm{AU}$ at the $6.0 \pm 1.0 \mathrm{pc}$ distance of this system. The peak flux of this source relative to that of WISE J0720-0846 corresponds to a relative magnitude of 4.1 $\pm 0.5 \mathrm{mag}$, consistent with that predicted from the SpeX spectral analysis, albeit with considerable uncertainty. This source is therefore a promising candidate for the brown dwarf companion, although both its validity and physical association with WISE J0720-0846 must be confirmed.

\subsection{Limits on the Companion Orbit from Radial Velocity Monitoring}

We can constrain the presence of an unresolved companion from the radial velocity measurements obtained over 3 months with Keck/NIRSPEC and over 2 months with Lick/Hamilton Spectrograph. In both sets of data, we find the measured radial velocities are constant within the uncertainties. For the NIRSPEC data, we measure a $\chi^{2}=5.01$ for three degrees of freedom (dof), corresponding to a $17 \%$ false alarm probability (marginal signficance); for the Hamilton data, we measure a $\chi^{2}=5.21$ for six dof, corresponding to a $52 \%$ false alarm probability (no significance). The marginal significance in the NIRSPEC data may be indicative of an additional $<0.5 \mathrm{~km} \mathrm{~s}^{-1}$ systematic error not included in our forward modeling analysis (see Blake et al. 2008; Burgasser et al. 2012).

Using the simulations described in Appendix B, we ascertained the range of semimajor axes $(a)$ ruled out assuming no detection of radial velocity variability. Given the slight offset between the NIRSPEC and Hamilton measurements, we treated the data sets separately. We first converted the component spectral types to $T_{\text {eff }} \mathrm{s}$ using the empirical relations of Stephens et al. (2009), and then converted these to masses using the evolutionary models of Burrows et al. (2001) for ages 0.5-10 Gyr (Table 6). The NIRSPEC measurements rule out the presence of a companion with $a<0.46-0.76 \mathrm{AU}$ $(0.26-0.44 \mathrm{AU})$ at the $50 \%(80 \%)$ confidence level, the range reflecting young to old ages (Figure 14, Table 6). The Hamilton data, given its shorter time coverage, provides a less stringent constraint. Note that even the closest separations cannot be completely ruled out given the possibilities of a faceon orbit, an eccentric orbit observed at apoapse, or poor synching of observations and orbital inflection points.

These limits are consistent with the location of the possible resolved companion. We performed a second set of simulations that used both the radial velocity measurements and the observed separation to assess the distributions of probable semimajor axes, periods and primary radial velocity variability amplitudes. These are constrained to be in the range $0.77-1.4 \mathrm{AU}, 1.7-4.3 \mathrm{yr}$ and $1.2-5.0 \mathrm{~km} \mathrm{~s}^{-1}$, respectively. Both period and primary radial velocity amplitude are sensitive 

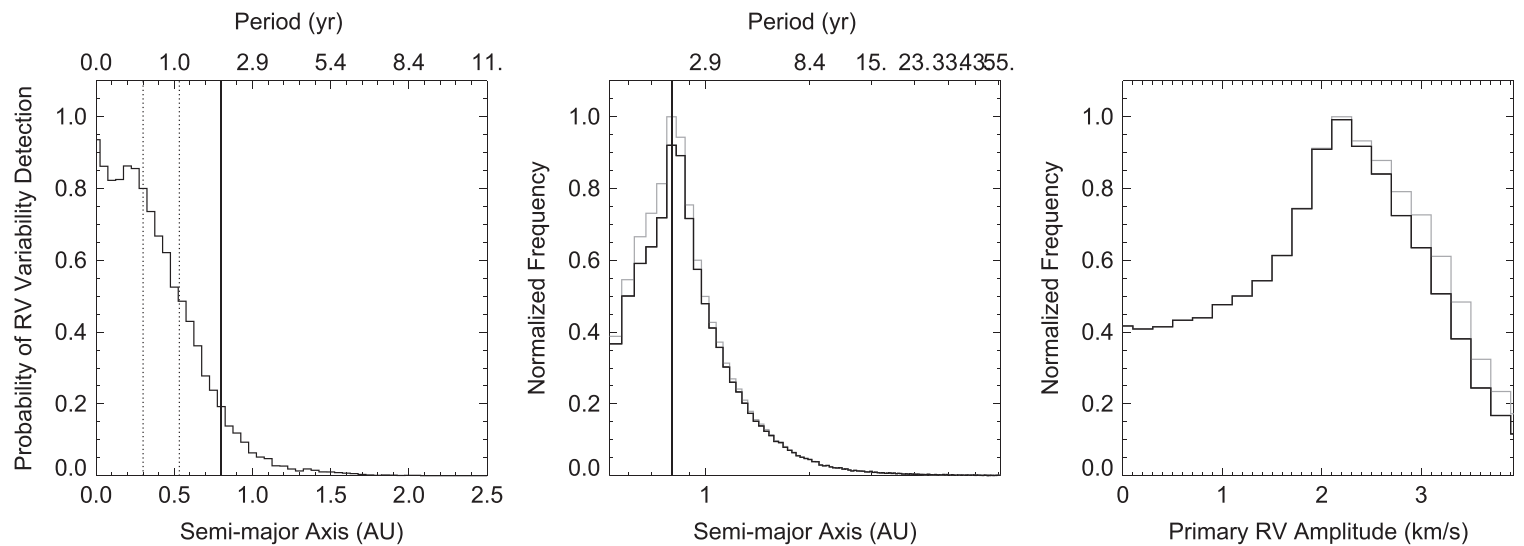

Figure 14. Results of orbit simulations for the WISE J0720-0846AB system based on the NIRSPEC radial velocity measurements and possible detection in NIRC2 images, assuming a system age of $1 \mathrm{Gyr}$. (Left) probability distribution of orbital semimajor axes based on the lack of variability in our radial velocity measurements. The dotted lines indicate the 80 and 50\% detection thresholds; the solid line indicates the most likely semimajor axis based on the putative NIRC2 detection ( 0.8 AU). (Center and right) probability distributions of semimajor axis (center) and primary radial velocity variability amplitude (right) based on the NIRC2 candidate detection alone (black histogram) and with the radial velocity constraints (gray histogram).

to the age and component masses of the system. Given the reasonably short time scale, maximum angular separation $(0,13-0.23)$, and significant radial velocity perturbations, this system is an excellent target for individual component mass measurements in the near term.

\section{DISCUSSION}

\subsection{Physical Properties of the WISE J0720-0846 System}

The inferred properties of WISE J0720-0846 are summarized in Table 5. Based on the analysis described above, we conclude that WISE J0720-0846A is a relatively old, magnetically active, low-mass field star. Its degree of activity (e.g., flaring rate) and its rotation frequency are both somewhat below those of other late-type M dwarfs, suggesting some longterm angular momentum loss may have occurred for this source. The putative T dwarf companion WISE J0720-0846B must be substellar, and its time-dependent cooling permits a model-dependent constraint on the age of the system if the component masses can be determined. We can already estimate that the mass ratio of this system $q \equiv \mathrm{M}_{2} / \mathrm{M}_{1}$ is highly agedependent, with $0.47 \leqslant q \leqslant 0.87$ for $1 \leqslant \tau \leqslant 10$ Gyr. If the possible source detected in our NIRC2 images is the companion, an inertial orbit measurement should be achieveable in $2-5 \mathrm{yr}$ by combining astrometric and radial velocity monitoring. Note that a robust detection of photometric variability would allow us to measure the rotational axis inclination of WISE J0720-0846A and assess spin-orbit alignment in this system, a critical test of binary formation that has only been examined in one VLM binary to date (Harding et al. 2013).

It is worth noting the striking similarities between WISE J0720-0846AB and two other late-M plus T spectral binaries SDSS J0006-0852AB and 2MASS J0320-0446AB, both M8.5 + T5 systems. The common classification of the secondary in all of these systems is likely a selection bias, as this subtype lies at the peak of the so-called "J-band bump," the 1 $\mu \mathrm{m}$ brightening from late-L to mid-T likely caused by the depletion of photospheric clouds at the $\mathrm{L}$ dwarf/T dwarf transition (Ackerman \& Marley 2001; Burgasser et al. 2002, 2013; Gelino et al. 2008; Marley et al. 2010). T5 companions are simply more readily detectable in blended light
Table 5

Dervied Properties of the WISE J0720-0846AB System

\begin{tabular}{lc}
\hline \hline Parameter & Value \\
\hline$\alpha(\mathrm{J} 2000)^{\mathrm{a}}$ & $07^{\mathrm{h}} 20^{\mathrm{m}} 02^{\mathrm{s}} .19$ \\
$\delta(\mathrm{J} 2000)^{\mathrm{a}}$ & $-08^{\circ} 46^{\prime} 59.53$ \\
Optical SpT & $\mathrm{M} 9.5$ \\
$\mathrm{NIR} \mathrm{SpT}$ & $\mathrm{M} 9+\mathrm{T} 5$ \\
$\pi(\mathrm{mas})$ & $166 \pm 28$ \\
$d(\mathrm{pc})$ & $6.0 \pm 1.0$ \\
$\mu_{\alpha}\left(\mathrm{mas} \mathrm{yr}^{-1}\right)$ & $-40.3 \pm 0.2$ \\
$\mu_{\delta}\left(\mathrm{mas} \mathrm{yr}^{-1}\right)$ & $-114.8 \pm 0.4$ \\
$V_{\mathrm{tan}}\left(\mathrm{km} \mathrm{s}^{-1}\right)$ & $3.5 \pm 0.6$ \\
$\mathrm{RV}\left(\mathrm{km} \mathrm{s}^{-1}\right)$ & $+83.1 \pm 0.4$ \\
$U\left(\mathrm{~km} \mathrm{~s}^{-1}\right)$ & $-47.5 \pm 0.4$ \\
$V\left(\mathrm{~km} \mathrm{~s}^{-1}\right)$ & $-47.6 \pm 0.4$ \\
$W\left(\mathrm{~km} \mathrm{~s}^{-1}\right)$ & $8.0 \pm 0.5$ \\
$v \sin i\left(\mathrm{~km} \mathrm{~s}^{-1}\right)$ & $8.0 \pm 0.5$ \\
$\left\langle\log _{10} L_{H \alpha} / L_{\mathrm{bol}}\right\rangle$ & $-4.68 \pm 0.06$ \\
$\Delta J^{\mathrm{b}}$ & $3.3 \pm 0.2$ \\
$\Delta H^{\mathrm{b}}$ & $4.1 \pm 0.4$ \\
$\Delta K^{\mathrm{b}}$ & $4.7 \pm 0.4$ \\
$\rho\left(\mathrm{mas}^{\mathrm{b}}\right)$ & $139 \pm 14$ \\
$\rho(\mathrm{AU})$ & $0.84 \pm 0.17$ \\
\hline
\end{tabular}

a At epoch 2014 January 1 (UT).

${ }^{b}$ Based on SpeX analysis, magnitudes are on the MKO system.

spectra due to their brighter magnitudes. All three systems also have separations $\lesssim 1 \mathrm{AU}$, below the $\sim 4-7 \mathrm{AU}$ peak of the separation distribution of resolved VLM binaries (Allen 2007). This agreement supports evidence that the spectral binary method is uncovering a significant population of tight binaries (Bardalez Gagliuffi et al. 2014). Finally, all three systems appear to be relatively mature. SDSS J0006-0852AB is $\gtrsim 7 \mathrm{Gyr}$ based on the inactivity of its widely separated M7 tertiary; 2MASS J0320-0446AB is $\gtrsim 2$ Gyr based on the mass and evolutionary state of its companion; WISE J0720-0846AB may be $\sim 5 \mathrm{Gyr}$ based on its old disk kinematics. This congruence may reflect the known preference for resolved VLM binaries to have nearly equal mass components (Bouy et al. 2003; Burgasser et al. 2003; Close et al. 2003; however, see below). A brown dwarf closer to the hydrogen burning minimum mass (HBMM) must cool longer to reach a $T_{\text {eff }} \approx 1200 \mathrm{~K}$ consistent 

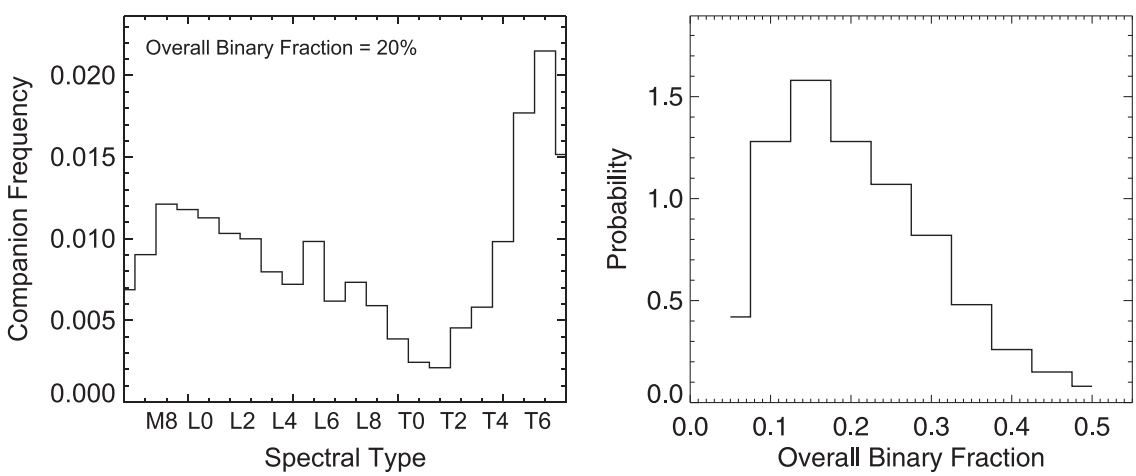

Figure 15. (Left): frequency distribution of companion spectral types for M7-M9.5 primaries based on the simulation described in Appendix C and assuming an overall binary fraction $\epsilon=20 \%$. Mid-type T dwarfs are far more common per subtype than late M and L dwarfs, and comprise 25\% of all companions. (Right): probability of finding two or more exclusively $\mathrm{T} 2-\mathrm{T} 7$ companions in a sample of 14 late $\mathrm{M}$ dwarfs as a function of overall binary fraction, based on these simulations. The distribution peaks at $1.6 \%$ for $\epsilon=15 \%$. The decline at smaller $\epsilon$ reflects the lack of companions; the decline of large $\epsilon$ results from contamination from earliertype companions.

Table 6

Constraints on the Companion Orbit from Radial Velocity and Imaging Measurements

\begin{tabular}{|c|c|c|c|c|c|c|c|c|}
\hline \multirow[b]{2}{*}{$\begin{array}{l}\text { System } \\
\text { Age } \\
(\mathrm{Gyr}) \\
\end{array}$} & \multirow[b]{2}{*}{$\begin{array}{c}\text { Primary }^{\mathrm{a}} \\
\text { Mass } \\
\left(M_{\odot}\right)\end{array}$} & \multirow[b]{2}{*}{$\begin{array}{c}\text { Secondary } \\
\text { Mass } \\
\left(M_{\odot}\right)\end{array}$} & \multirow[b]{2}{*}{$\begin{array}{l}\text { Mass } \\
\text { Ratio }\end{array}$} & \multicolumn{2}{|c|}{ NIRSPEC Only } & \multicolumn{3}{|c|}{ NIRSPEC + NIRC2 } \\
\hline & & & & $\begin{array}{c}\text { Detection } \\
\text { Limit }(80 \%) \\
(\mathrm{AU})\end{array}$ & $\begin{array}{c}\text { Detection } \\
\text { Limit }(50 \%) \\
(\mathrm{AU})\end{array}$ & $\begin{array}{c}\text { Semimajor } \\
\text { Axis Range } \\
\text { (AU) }\end{array}$ & $\begin{array}{c}\text { Period } \\
\text { Range } \\
(\mathrm{yr})\end{array}$ & $\begin{array}{c}\text { Primary } \\
\text { RV Range } \\
\left(\mathrm{km} \mathrm{s}^{-1}\right)\end{array}$ \\
\hline 0.5 & 0.066 & 0.027 & 0.41 & 0.26 & 0.46 & $0.77-1.2$ & $2.2-4.3$ & $1.2-2.4$ \\
\hline 1.0 & 0.077 & 0.036 & 0.47 & 0.30 & 0.53 & $0.78-1.3$ & $2.0-3.9$ & $1.4-2.9$ \\
\hline 5.0 & 0.084 & 0.065 & 0.77 & 0.41 & 0.72 & $0.77-1.2$ & $1.8-3.4$ & $2.2-4.6$ \\
\hline
\end{tabular}

${ }^{a}$ Based on the evolutionary models of Burrows et al. (2001), system ages listed, and component $T_{\text {eff }} \mathrm{s}$ of $2300 \mathrm{~K}$ and $1100 \mathrm{~K}$ for WISE J0720-0846A and B based on the $T_{\text {eff }} /$ spectral type relation of Stephens et al. (2009).

with a mid-T dwarf. From these few examples, we speculate that late-M dwarf plus $\mathrm{T}$ dwarf spectral binary systems specifically probe an old, tightly bound population of VLM binaries, which are particularly useful systems for orbital mass measurements.

\subsection{Late-M+T Dwarf Binaries in the Local Sample}

WISE J0720-0846 joins the M8.5 SCR 1845-6357AB system (Biller et al. 2006; Kasper et al. 2007) as one of two late-M plus $\mathrm{T}$ dwarf binaries in the immediate vicinity of the Sun. Remarkably, these are the only binary systems among the 14 M7-M9.5 dwarf primaries known within 10 pc (Table 7). This fact is particularly surprising given the numerous efforts to identify faint companions to cool stars close to the Sun (Bouy et al. 2003; Close et al. 2003; Martn et al. 2006; Dieterich et al. 2012), and the apparent preference for high mass-ratio systems among VLM binaries. As it stands, this volume-limited sample has a brown dwarf companion fraction, $\epsilon_{\mathrm{BD}}=14_{-5}^{+14} \%$, that is marginally higher (but consistent with) its stellar companion fraction limit $\epsilon_{*}<12 \% \quad(1 \sigma \quad$ binomial uncertainties).

Is the nature of multiples in the local late-M dwarf population simply due to small number statistics? To examine this question, we performed a population simulation similar to those described in Burgasser $(2004,2007 a)$, combined with a random draw experiment to determine the likelihood of various binary configurations; see Appendix $\mathrm{C}$ for details. From our simulations we find that $\mathrm{T}$ dwarfs are remarkably common companions to late $\mathrm{M}$ dwarfs, comprising $25 \%$ of secondaries
(Figure 15). Their relative abundance derives from two factors. First, the typical masses of M7-M9.5 dwarfs in our simulation, $0.089 \pm 0.003 M_{\odot}$, are close to the HBMM, so stellar companions exist over a relatively narrow range of masses. Brown dwarf companions to late-M primaries consistently outnumber stellar companions in our simulation; in contrast, only 9\% of systems with mid-M (M4-M7) primaries host T dwarf companions. Second, there is the well-known "pile-up" of T dwarfs in field brown dwarf populations due to their slow cooling rates (Burgasser 2004; Allen et al. 2005). T dwarfs are the most common companion to the oldest systems in our simulation ( $>5 \mathrm{Gyr}$ ). This is consistent with the old ages inferred for SDSS J0006-0852AB, 2MASS J0320-0446AB and WISE J0720-0846AB.

Even with the prevalence of $\mathrm{T}$ dwarf companions to late-M primaries, the incidence of finding 2 or more $\mathrm{T}$ dwarf companions in the nearby sample-and only $\mathrm{T}$ dwarf companions-remains low, peaking at $1.6 \%$ for a total binary fraction $\epsilon=15 \%$. The local sample would seem to be an anomaly if the underlying multiplicity distributions are accurately characterized. It is possible that more (and less) massive companions to other late $\mathbf{M}$ dwarfs in the nearby sample have yet to be found, and may be uncovered astrometrically with the current GAIA mission (Perryman et al. 2001; Zwitter \& Munari 2004). We also note that while this specific configuration is rare, there are roughly $10^{8} 10 \mathrm{pc}$ "bubbles" in the 3-12 kpc Galactic disk, making our sample of M dwarfs one of a million.

Finally, we note that the peak probability of exclusively $\mathrm{T}$ dwarf companions to late $\mathrm{M}$ dwarfs occurs in a range of $\epsilon$ 
Table 7

M7-M9.5 Dwarf Primaries within 10 pc

\begin{tabular}{|c|c|c|c|c|c|}
\hline Source & $\mathrm{SpT}$ & $\begin{array}{c}\pi \\
(\mathrm{mas})\end{array}$ & $M_{K}$ & $V-K$ & Ref. \\
\hline SCR 1845-6357AB & $\mathrm{M} 8.5+\mathrm{T} 6$ & $259.5 \pm 1.1$ & 10.58 & 8.89 & 1,10 \\
\hline DENIS J1048-3956 & M8.5 & $249.8 \pm 1.8$ & 10.44 & 8.88 & 1 \\
\hline 2MASS J1540-5101 & M7 & $228 \pm 24$ & 9.75 & 7.32 & 17 \\
\hline LSR J1835 + 3259 & M8.5 & $176.5 \pm 0.5$ & 10.41 & 9.10 & 2 \\
\hline WISE J0720-0846AB & M9.5+T5 & $166 \pm 28$ & 10.5 & $\cdots$ & 3,4 \\
\hline LP 944-20 & M9 & $155.9 \pm 1.0$ & 10.51 & 9.15 & 5 \\
\hline GJ 3877 & M7 & $152 \pm 2$ & 9.84 & 8.02 & 5,7 \\
\hline SCR 1546-5534 & M8: & $149 \pm 40$ & 10.0 & $\cdots$ & 6 \\
\hline SIPS 1259-4336 & M8: & $\sim 128$ & $\sim 10.1$ & $\cdots$ & 9,11 \\
\hline LEHPM 1-3396 & M9 & $121 \pm 4$ & 10.80 & $\cdots$ & 12,13 \\
\hline LHS 2065 & M9 & $118.0 \pm 0.8$ & 10.30 & 9.00 & 5,7 \\
\hline 1RXS J1159-5247 & M9 & $105.54 \pm 0.12$ & 10.44 & $\ldots$ & 14,15 \\
\hline LP $655-48$ & M7 & $106 \pm 3$ & 9.66 & 8.31 & 1,16 \\
\hline LP 647-13 & M9 & $104 \pm 2$ & 10.52 & 8.84 & 8 \\
\hline
\end{tabular}

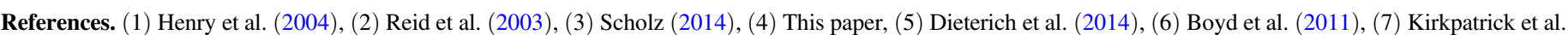

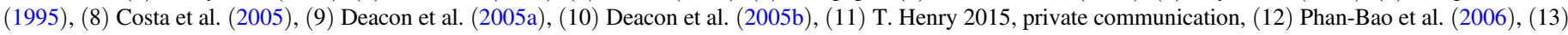
Faherty et al. (2012), (14) Hambaryan et al. (2004), (15) Sahlmann et al. (2014), (16) Shkolnik et al. (2012), (17) Pérez Garrido et al. (2014).

consistent with previous determinations of the VLM binary fraction (Siegler et al. 2005; Basri \& Reiners 2006; Burgasser et al. 2006; Allen 2007). This is significant, as the decline in frequency at high fractions is caused by increased contamination by earlier-type secondaries. The lack of such companions in the nearby sample may be a consequence of the low binary fraction of VLM dwarfs compared to more massive stars. The companionship of late-M dwarfs appears to be determined by the confluence of four key statistics of VLM dwarfs: the mass function across the substellar limit, the cooling rate of brown dwarfs, the mass ratio distribution of VLM dwarfs, and the underlying multiplicity fraction. The importance of these statistics for brown dwarf formation and interior theories motivates a complete assessment of the multiplicity properties of late $\mathrm{M}$ dwarfs in a much larger volume.

\section{SUMMARY}

We have conducted a detailed investigation of the recently identified, nearby VLM dwarf WISE J0720-0846. From optical and infrared imaging and spectroscopic investigations, we have determined the optical and near-infrared classifications of this source, improved its astrometry, measured its spatial kinematics and rotational velocity, identified persistent and flaring magnetic activity, and found evidence for a brown dwarf companion. The reduced level of activity in this source compared to other late-M dwarfs, and its old disk kinematics, both suggest that this is a relatively mature system. The putative $\mathrm{T}$ dwarf companion, identified in combined-light spectroscopy and possibly resolved at $1 \mathrm{AU}$ projected separation, will aid in constraining the age, as astrometric and radial velocity monitoring over the next few years should allow us to map the orbit of the system and extract individual component masses. Remarkably, this is one of only two binaries among late $\mathrm{M}$ dwarfs in the immediate vicinity of the Sun, and both have $\mathrm{T}$ dwarf companions. We argue that, while rare, this may reflect a combination of the proximity of the primary to the HBMM, the evolutionary properties of brown dwarfs, and the underlying binary fraction, making multiplicity studies of late
M dwarfs a potentially useful window into brown dwarf formation and evolution theories.

The authors thank Bill Golisch and John Rayner at IRTF; Wayne Earthman, Erik Kovacs, Donnie Redel and Pavl Zachary at Lick Observatory; Diane Harmer and Krissy Reetz at KPNO; Scott Dahm, Greg Doppmann, Heather Hershley, Gary Punawai, Luca Rizzi, and Terry Stickel at Keck for their assistance with the observations. We also acknowledge useful discussions with Gregg Hallinan and Stuart P. Littlefair on M dwarf magnetic activity; and John Gizis, Todd Henry, J. Davy Kirkpatrick, Nicholas Lodieu, and I. Neill Reid on the 10 pc sample. We thank our referee, R. Scholz, for his very helpful comments that allowed us to considerably improve the manuscript. This research has made use of the SIMBAD database, operated at CDS, Strasbourg, France; the M, L, T, and Y dwarf compendium housed at DwarfArchives.org; and the SpeX Prism Spectral Libraries at http://www.browndwarfs. org/spexprism. C. M. acknowledges support from the National Science Foundation under award No. AST-1313428. TRAPPIST is a project funded by the Belgian Fund for Scientific Research (F.R.S.-FNRS) under grant FRFC 2.5.594.09.F, with the participation of the Swiss National Science Foundation. M. Gillon and E. Jehin are F.R.S.-FNRS Research Associates. L. Delrez and J. Manfroid acknowledge the support of the F.R. S.-FNRS for their PhD theses. The authors wish to recognize and acknowledge the very significant cultural role and reverence that the summit of Mauna Kea has always had within the indigenous Hawaiian community. We are most fortunate to have the opportunity to conduct observations from this mountain.

Note added in proof. Shortly after acceptance of this publication, Ivanov et al. (2015) reported their own observations of WISE J07200846. They find a formally consistent spectral classification ( $\mathrm{L} 0 \pm 1)$, radial velocity $\left(79 \pm 3 \mathrm{~km} \mathrm{~s}^{-1}\right)$, and parallactic distance $\left(6.1_{-1.0}^{+1.4} \mathrm{pc}\right)$. 
They did not acquire high resolution images sufficient to confirm or refute the companion candidate reported here.

\section{APPENDIX A PDM ANALYSIS}

The PDM technique identifies periodic signals in time-series data by searching for minimal dispersion about a mean phased signal (Stellingwerf 1978). The frequency sampling is set by the fundamental frequency $f_{1} \equiv 1 / T=0.021$ cycles day $^{-1}$, where $T=1160 \mathrm{hr}$ is the full monitoring period (Scargle 1982; Press \& Teukolsky 1988). We examined 1081 periods in the period range $4 \mathrm{hr}<P<16 \mathrm{hr}$, sampled evenly in frequency space in steps of $f_{1} / 10$. For each period, we phase-folded the light curve, computed a mean curve sampled at $r=100$ linearly spaced phase points, then computed the $\chi^{2}$ deviation of the phased data relative to the mean curve. The statistic of merit is the ratio

$$
\Theta(P)=\frac{\chi^{2}(P)}{\chi_{o}^{2}} \frac{N-1}{N-r}
$$

where $\chi_{o}^{2}$ is measured from the unphased light curve, and $N=5895$ is the total number of data points.

Following Schwarzenberg-Czerny (1997), we assessed the significance of minima in $\Theta(P)$ using the regularized incomplete beta function $I$ :

$$
\begin{aligned}
\operatorname{Pr}(\Theta<z)= & {\left[I_{\frac{N-r}{N-1} z}\left(\frac{N-r}{2}, \frac{r-1}{2}\right) /\right.} \\
& \left.I_{1}\left(\frac{N-r}{2}, \frac{r-1}{2}\right)\right]^{1 / N_{f}} .
\end{aligned}
$$

Here, $\operatorname{Pr}(\Theta<z)$ is the false-alarm probability when $\Theta$ is less than some value $0<z<1$ by chance; 1 -Pr gives the significance of a period corresponding to a minimum in $\Theta$. The $1 / N_{f}$ exponent is the band penalty incurred when searching for multiple periods, where we have used

$$
N_{f}=2 \frac{f_{\text {high }}-f_{\text {low }}}{f_{1}}=432
$$

as the number of independent frequencies sampled (Press \& Teukolsky 1988). Periods with 1-Pr $>90 \%$ were deemed significant, but no features in the cleaned and normalized light curve satisfied this limit.

\section{APPENDIX B \\ SIMULATIONS FOR ORBIT CONSTRAINTS}

To quantify the companion detection limits from our radial velocity observations, and determine whether the candidate companion identified in NIRC2 imaging data is consistent with these limits, we performed a pair of Monte Carlo orbit simulations. The first simulation aimed to determine the range of orbital semimajor axes that would have been detected given the radial velocity measurement uncertainties and sampling. We generated a large number $\left(10^{6}\right)$ of hypothetical orbits, uniformly sampling semimajor axes $0<a<2.5 \mathrm{AU}$, eccentricities $0<\epsilon<0.6$ (Dupuy \& Liu 2011), sin inclinations $0<\sin i<1$, longitude of ascending node $0<\Omega<2 \pi$, argument of periapse $0<\omega<2 \pi$, and mean anomaly angle $0<M_{\text {NIRC2 }}<2 \pi$ for the NIRC2 imaging epoch. We then solved for the maximum radial velocity amplitude of the primary component (e.g., Ford 2005):

$$
V_{1}=\frac{2 \pi a \sin i}{P \sqrt{1-\epsilon^{2}}} \frac{M_{2}}{M_{1}+M_{2}},
$$

with component masses $M_{1}$ and $M_{2}$ estimated from the evolutionary models of Burrows et al. (2001), system ages of $0.5,1,5$ and $10 \mathrm{Gyr}$, and component $T_{\text {eff }} \mathrm{s}$ of 2300 and $1100 \mathrm{~K}$ for WISE J0720-0846A and B based on the $T_{\text {eff }} /$ spectral type relation of Stephens et al. (2009) (Table 6). The observed radial motion of the primary at each NIRSPEC or Hamilton epoch $t_{i}$ (these data sets were modeled separately) was then calculated as

$$
v\left(t_{i}\right)=V_{1}\left(\epsilon \cos \omega+\cos \left(T\left(t_{i}\right)-\omega\right)\right)
$$

where

$$
\tan \frac{T\left(t_{i}\right)}{2}=\sqrt{\frac{1+\epsilon}{1-\epsilon}} \tan \frac{E\left(t_{i}\right)}{2}
$$

relates the true anomaly $T$ to the eccentric anomaly $E$, which is in turn related to the mean anomaly through Kepler's equation:

$$
M\left(t_{i}\right)-M_{0}=2 \pi \frac{t_{i}-\tau_{0}}{P}=E-\epsilon \sin E .
$$

Here, $P$ is the period of the orbit, determined from the estimated masses, input semimajor axis, and Kepler's Period Law; and $t_{i}-\tau_{0}$ is the time since periastron passage. Setting $M_{0}=0$ and $t_{\mathrm{NIRC} 2}=0$ (the time of the NIRC2 image), we can set $\tau_{0}=-\mathrm{PM}_{\mathrm{IRC} 2} / 2 \pi$ and solve for the primary velocities numerically.

For each simulated orbit, we calculated $\chi^{2}$ for the radial velocity epochs relative to their mean, using the corresponding observational uncertainties. We then determined the fraction of orbits that exceeded our measured $\chi^{2}$ as a function of semimajor axis. Figure 14 displays the results of this calculation for the NIRSPEC data and a system age of 1 Gyr. Due to inclination variations and sampling, detection probabilities never reach 100\%; we therefore use 50 and $80 \%$ probabilities of detection as our thresholds.

To add in the constraint of a possible detection of the companion at a projected separation of $\rho=0$." 14 , we used the same orbital parameters to assess the range of semimajor axes consistent with this separation. Cartesian positions in the plane of the sky for an orbit of unit semimajor axis are

$$
\begin{aligned}
& x=A(\cos E-\epsilon)+F \sqrt{1-\epsilon^{2}} \sin E \\
& y=B(\cos E-\epsilon)+G \sqrt{1-\epsilon^{2}} \sin E
\end{aligned}
$$

were $A, B, F$ and $G$ are the Thiele-Innes constants (Innes 1907; van den Bos 1927):

$$
\begin{gathered}
A=\cos \omega \cos \Omega-\sin \omega \sin \Omega \cos i \\
B=\cos \omega \sin \Omega+\sin \omega \cos \Omega \cos i \\
F=-\sin \omega \cos \Omega-\cos \omega \sin \Omega \cos i \\
G=-\sin \omega \sin \Omega+\cos \omega \cos \Omega \cos i .
\end{gathered}
$$

The total projected separation $\Delta=\sqrt{x^{2}+y^{2}}$ can then be compared to the observed projected separation $(\rho d)$ to constrain the semimajor axis for a given orbit $i$ : 


$$
a_{i}=\frac{\rho d}{\Delta_{i}} .
$$

Figure 14 displays the distributions of semimajor axes and primary radial velocity variability amplitudes consistent both with the possible detection and lack of detectable radial velocity variation.

\section{APPENDIX C \\ SIMULATION FOR LOCAL BINARY STATISTICS}

We performed another Monte Carlo simulation to assess the likelihood of finding two or more $\mathrm{T}$ dwarf companions (exclusively) in the sample of 14 M7-M9.5 dwarfs within 10 pc of the Sun (Table 7). We started with a sample of $N=10^{5}$ primaries with uniform ages spanning 0.5-7 Gyr (corresponding to the typical ages of disk stars) and masses $0.013 M_{\odot} \leqslant M$ $\leqslant 0.2 M_{\odot}$ distributed as a power-law mass function $d N / d M \propto M^{-0.5}$ (Reid et al. 1999; Kirkpatrick et al. 2012; Burningham et al. 2013). For each system we assigned a secondary mass using the power-law mass ratio distribution of Allen (2007), $d N / d q \propto q^{1.8}$, based on Bayesian analysis of VLM imaging samples; this distribution favors equal-mass systems. The component masses and system ages were transformed to bolometric luminosities using the evolutionary models of Burrows et al. (2001), and these converted into spectral types by combining the spectral type/absolute $J$-band magnitude relation of Dupuy \& Liu (2012) with the spectral type/J-band bolometric correction relation of Liu et al. (2010). Systems with component spectral types outside the range M5T9 were rejected.

We selected a subset of M7-M9.5 sources (4626 systems) and used these as the primaries for our random-draw experiment. The distribution of companion types for this sample is shown in Figure 15. Flagging a randomly-assigned subset $N_{b}=\epsilon N$ of these systems as actual binaries, with $\epsilon \in$ $[0.05,0.5]$, we repeatedly $\left(10^{5}\right.$ times $)$ drew 14 systems from this collection and determined the fraction that contained at least two companions with spectral types T2-T7 (detectable by the spectral binary method; Burgasser 2007b) and no other companion types. Figure 15 displays the frequency of this outcome as a function of overall binary fraction.

\section{REFERENCES}

Ackerman, A. S., \& Marley, M. S. 2001, ApJ, 556, 872

Allard, F., Homeier, D., \& Freytag, B. 2012, in ASP Conf. Ser. 448, 16th Cambridge Workshop on Cool Stars, Stellar Systems, and the Sun, ed. C. M. Johns-Krull, M. K. Browning, \& A. A. West (San Francisco, CA: ASP), 91

Allen, P. R. 2007, ApJ, 668, 492

Allen, P. R., Koerner, D. W., Reid, I. N., \& Trilling, D. E. 2005, ApJ, 625, 385

Artigau, É, Radigan, J., Folkes, S., et al. 2010, ApJL, 718, L38

Bailer-Jones, C. A. L., \& Lamm, M. 2003, MNRAS, 339, 477

Bardalez Gagliuffi, D. C., Burgasser, A. J., Gelino, C. R., et al. 2014, ApJ, 794, 143

Basri, G., \& Reiners, A. 2006, AJ, 132, 663

Bihain, G., Scholz, R.-D., Storm, J., \& Schnurr, O. 2013, A\&A, 557, A43

Bildsten, L., Brown, E. F., Matzner, C. D., \& Ushomirsky, G. 1997, ApJ, 482, 442

Biller, B. A., Kasper, M., Close, L. M., Brandner, W., \& Kellner, S. 2006, ApJL, 641, L141

Blake, C. H., Charbonneau, D., \& White, R. J. 2010, ApJ, 723, 684

Blake, C. H., Charbonneau, D., White, R. J., et al. 2008, ApJL, 678, L125

Bochanski, J. J., West, A. A., Hawley, S. L., \& Covey, K. R. 2007, AJ, 133, 531

Bouy, H., Brandner, W., Martín, E. L., et al. 2003, AJ, 126, 1526
Boyd, M. R., Henry, T. J., Jao, W.-C., Subasavage, J. P., \& Hambly, N. C. 2011, AJ, 142, 92

Burgasser, A. J. 2004, ApJS, 155, 191

Burgasser, A. J. 2007, ApJ, 659, 655

Burgasser, A. J. 2007, AJ, 134, 1330

Burgasser, A. J. 2014, in Proc. ASI Conf. Ser. 11, 2013 Int. Workshop on Spectral Stellar Libraries, ed. H. P. Singh, P. Prugniel, \& I. Vauglin, 7

Burgasser, A. J., Cruz, K. L., Cushing, M., et al. 2010, ApJ, 710, 1142

Burgasser, A. J., Kirkpatrick, J. D., Cruz, K. L., et al. 2006, ApJS, 166, 585

Burgasser, A. J., Kirkpatrick, J. D., Reid, I. N., et al. 2003, ApJ, 586, 512

Burgasser, A. J., Liu, M. C., Ireland, M. J., Cruz, K. L., \& Dupuy, T. J. 2008, ApJ, 681, 579

Burgasser, A. J., Luk, C., Dhital, S., et al. 2012, ApJ, 757, 110

Burgasser, A. J., Marley, M. S., Ackerman, A. S., et al. 2002, ApJL, 571, L151

Burgasser, A. J., \& McElwain, M. W. 2006, AJ, 131, 1007

Burgasser, A. J., McElwain, M. W., Kirkpatrick, J. D., et al. 2004, AJ, 127,2856

Burgasser, A. J., Sheppard, S. S., \& Luhman, K. L. 2013, ApJ, 772, 129

Burgasser, A. J., Sitarski, B. N., Gelino, C. R., Logsdon, S. E., \& Perrin, M. D. 2011, ApJ, 739, 49

Burgasser, A. J., Witte, S., Helling, C., et al. 2009, ApJ, 697, 148

Burningham, B., Cardoso, C. V., Smith, L., et al. 2013, MNRAS, 433, 457

Burrows, A., Hubbard, W. B., Lunine, J. I., \& Liebert, J. 2001, RvMP, 73, 719 Churchill, C. W. 1995, Lick Technical Report, 74

Close, L. M., Siegler, N., Freed, M., \& Biller, B. 2003, ApJ, 587, 407

Costa, E., Méndez, R. A., Jao, W.-C., et al. 2005, AJ, 130, 337

Cruz, K. L., Burgasser, A. J., Reid, I. N., \& Liebert, J. 2004, ApJL, 604, L61

Cruz, K. L., Kirkpatrick, J. D., \& Burgasser, A. J. 2009, AJ, 137, 3345

Cruz, K. L., Reid, I. N., Kirkpatrick, J. D., et al. 2007, AJ, 133, 439

Cushing, M. C., Kirkpatrick, J. D., Gelino, C. R., et al. 2011, ApJ, 743, 50

Cushing, M. C., Vacca, W. D., \& Rayner, J. T. 2004, PASP, 116, 362

Davies, S. R. 1990, MNRAS, 244, 93

Day-Jones, A. C., Marocco, F., Pinfield, D. J., et al. 2013, MNRAS, 430, 1171

Deacon, N. R., Hambly, N. C., \& Cooke, J. A. 2005, A\&A, 435, 363

Deacon, N. R., Hambly, N. C., Henry, T. J., et al. 2005, AJ, 129, 409

Deacon, N. R., Liu, M. C., Magnier, E. A., et al. 2014, ApJ, 792, 119

Dieterich, S. B., Henry, T. J., Golimowski, D. A., Krist, J. E., \& Tanner, A. M. 2012, AJ, 144, 64

Dieterich, S. B., Henry, T. J., Jao, W.-C., et al. 2014, AJ, 147, 94

Dupuy, T. J., \& Liu, M. C. 2011, ApJ, 733, 122

Dupuy, T. J., \& Liu, M. C. 2012, ApJS, 201, 19

Eggen, O. J. 1969, PASP, 81, 553

Faherty, J. K., Burgasser, A. J., Walter, F. M., et al. 2012, ApJ, 752, 56

Ford, E. B. 2005, AJ, 129, 1706

Gelino, C. R., \& Burgasser, A. J. 2010, AJ, 140, 110

Gillon, M., Triaud, A. H. M. J., Jehin, E., et al. 2013, A\&A, 555, L5

Gizis, J. E., Monet, D. G., Reid, I. N., et al. 2000, AJ, 120, 1085

Hambaryan, V., Staude, A., Schwope, A. D., et al. 2004, A\&A, 415, 265

Hambly, N. C., Davenhall, A. C., Irwin, M. J., \& MacGillivray, H. T. 2001, MNRAS, 326, 1315

Hambly, N. C., Henry, T. J., Subasavage, J. P., Brown, M. A., \& Jao, W.-C. 2004, AJ, 128, 437

Hambly, N. C., Irwin, M. J., \& MacGillivray, H. T. 2001, MNRAS, 326, 1295 Hambly, N. C., MacGillivray, H. T., Read, M. A., et al. 2001, MNRAS, 326, 1279

Hamuy, M., Walker, A. R., Suntzeff, N. B., et al. 1992, PASP, 104, 533

Harding, L. K., Hallinan, G., Konopacky, Q. M., et al. 2013, A\&A, 554, A113

Henry, T. J., Jao, W.-C., Subasavage, J. P., et al. 2006, AJ, 132, 2360

Henry, T. J., Subasavage, J. P., Brown, M. A., et al. 2004, AJ, 128, 2460

Hilton, E. J., West, A. A., Hawley, S. L., \& Kowalski, A. F. 2010, AJ, 140,1402

Innes, R. T. A. 1907, Obs, 30, 310

Irwin, J., Berta, Z. K., Burke, C. J., et al. 2011, ApJ, 727, 56

Ivanov, V. D., Vaisanen, P., Kniazev, A. Y., et al. 2015, A\&A, in press, arXiv: 1410.6792

Jehin, E., Gillon, M., Queloz, D., et al. 2011, Msngr, 145, 2

Kasper, M., Biller, B. A., Burrows, A., et al. 2007, A\&A, 471, 655

Kirkpatrick, J. D., Cruz, K. L., Barman, T. S., et al. 2008, ApJ, 689, 1295

Kirkpatrick, J. D., Cushing, M. C., Gelino, C. R., et al. 2011, ApJS, 197, 19

Kirkpatrick, J. D., Gelino, C. R., Cushing, M. C., et al. 2012, ApJ, 753, 156

Kirkpatrick, J. D., Henry, T. J., \& Simons, D. A. 1995, AJ, 109, 797

Kirkpatrick, J. D., Reid, I. N., Liebert, J., et al. 1999, ApJ, 519, 802

Kirkpatrick, J. D., Schneider, A., Fajardo-Acosta, S., et al. 2014, ApJ, 783, 122

Leggett, S. K. 1992, ApJS, 82, 351

Lépine, S. 2005, AJ, 130, 1680

Lépine, S., Rich, R. M., \& Shara, M. M. 2003, AJ, 125, 1598 
Lépine, S., Rich, R. M., \& Shara, M. M. 2007, ApJ, 669, 1235

Liu, M. C., Dupuy, T. J., \& Leggett, S. K. 2010, ApJ, 722, 311

Livingston, W., \& Wallace, L. 1991, An atlas of the solar spectrum in the infrared from 1850 to $9000 \mathrm{~cm}-1$ ( 1.1 to 5.4 micrometer)

Lomb, N. R. 1976, Ap\&SS, 39, 447

Looper, D. L., Gelino, C. R., Burgasser, A. J., \& Kirkpatrick, J. D. 2008, ApJ, 685,1183

Looper, D. L., Kirkpatrick, J. D., \& Burgasser, A. J. 2007, AJ, 134, 1162

Lucas, P. W., Tinney, C. G., Burningham, B., et al. 2010, MNRAS, 408, L56

Luhman, K. L. 2013, ApJL, 767, L1

Luhman, K. L. 2014, ApJL, 786, L18

Marley, M. S., Saumon, D., \& Goldblatt, C. 2010, ApJL, 723, L117

Martín, E. L., Delfosse, X., Basri, G., et al. 1999, AJ, 118, 2466

Martín, E. L., Guenther, E., Zapatero Osorio, M. R., Bouy, H., \& Wainscoat, R. 2006, ApJL, 644, L75

McLean, I. S., Graham, J. R., Becklin, E. E., et al. 2000, Proc. SPIE, 4008, 1048

McLean, M., Berger, E., \& Reiners, A. 2012, ApJ, 746, 23

Mohanty, S., \& Basri, G. 2003, ApJ, 583, 451

Nidever, D. L., Marcy, G. W., Butler, R. P., Fischer, D. A., \& Vogt, S. S. 2002, ApJS, 141, 503

Pakhomov, Y. V., \& Zhao, G. 2013, AJ, 146, 97

Parker, Q. A., Phillipps, S., Pierce, M. J., et al. 2005, MNRAS, 362, 689

Perryman, M. A. C., de Boer, K. S., Gilmore, G., et al. 2001, A\&A, 369, 339

Phan-Bao, N., Bessell, M. S., Martín, E. L., et al. 2006, MNRAS, 366, L40

Pravdo, S. H., Shaklan, S. B., Wiktorowicz, S. J., et al. 2006, ApJ, 649, 389

Press, W. H., \& Teukolsky, S. A. 1988, ComPh, 2, 77

Probst, R. G., \& Liebert, J. 1983, ApJ, 274, 245

Rayner, J. T., Toomey, D. W., Onaka, P. M., et al. 2003, PASP, 115, 362

Reid, I. N., Cruz, K. L., Allen, P., et al. 2004, AJ, 128, 463

Reid, I. N., Cruz, K. L., Kirkpatrick, J. D., et al. 2008, AJ, 136, 1290

Reid, I. N., Cruz, K. L., Laurie, S. P., et al. 2003, AJ, 125, 354

Reid, I. N., Gizis, J. E., \& Hawley, S. L. 2002, AJ, 124, 2721

Reid, I. N., Hawley, S. L., \& Gizis, J. E. 1995, AJ, 110, 1838
Reid, I. N., Kirkpatrick, J. D., Liebert, J., et al. 1999, ApJ, 521, 613

Reiners, A., \& Basri, G. 2008, ApJ, 684, 1390

Reiners, A., \& Basri, G. 2009, ApJ, 705, 1416

Roeser, S., Demleitner, M., \& Schilbach, E. 2010, AJ, 139, 2440

Sahlmann, J., Lazorenko, P. F., Ségransan, D., et al. 2014, A\&A, 565, A20

Scargle, J. D. 1982, ApJ, 263, 835

Schmidt, S. J., Cruz, K. L., Bongiorno, B. J., Liebert, J., \& Reid, I. N. 2007, AJ, 133, 2258

Scholz, R.-D. 2014, A\&A, 561, A113

Scholz, R.-D., Bihain, G., Schnurr, O., \& Storm, J. 2011, A\&A, 532, L5

Scholz, R.-D., Lo Curto, G., Méndez, R. A., et al. 2005, A\&A, 439, 1127

Schönrich, R., Binney, J., \& Dehnen, W. 2010, MNRAS, 403, 1829

Schwarzenberg-Czerny, A. 1997, ApJ, 489, 941

Shkolnik, E. L., Anglada-Escudé, G., Liu, M. C., et al. 2012, ApJ, 758, 56

Siegler, N., Close, L. M., Cruz, K. L., Martín, E. L., \& Reid, I. N. 2005, ApJ, 621,1023

Simons, D. A., \& Tokunaga, A. 2002, PASP, 114, 169

Stellingwerf, R. F. 1978, ApJ, 224, 953

Stephens, D. C., Leggett, S. K., Cushing, M. C., et al. 2009, ApJ, 702, 154

Stetson, P. B. 1987, PASP, 99, 191

Teegarden, B. J., Pravdo, S. H., Hicks, M., et al. 2003, ApJL, 589, L51

Tody, D. 1986, Proc. SPIE, 627, 733

Tokunaga, A. T., Simons, D. A., \& Vacca, W. D. 2002, PASP, 114, 180

Vacca, W. D., Cushing, M. C., \& Rayner, J. T. 2003, PASP, 115, 389

van Dam, M. A., Bouchez, A. H., Le Mignant, D., et al. 2006, PASP, 118, 310

van den Bos, W. H. 1927, BAN, 3, 261

Vogt, S. S. 1987, PASP, 99, 1214

Walkowicz, L. M., Hawley, S. L., \& West, A. A. 2004, PASP, 116, 1105

West, A. A., Morgan, D. P., Bochanski, J. J., et al. 2011, AJ, 141, 97

Wizinowich, P. L., Le Mignant, D., Bouchez, A. H., et al. 2006, PASP, 118,297

Zwitter, T., \& Munari, U. 2004, in RMxAA Conf. Ser., Proc. IAU Colloq. 191, The Environment and Evolution of Double and Multiple Stars 21, ed. C. Allen, \& C. Scarfe, 251 